\title{
LONG TIME VERSUS STEADY STATE OPTIMAL CONTROL
}

\author{
ALESSIO PORRETTA AND ENRIQUE ZUAZUA
}

\begin{abstract}
This paper is devoted to analyze the convergence of optimal control problems for an evolution equation in a finite time-horizon $[0, T]$ towards the limit steady state ones as $T \rightarrow \infty$. We focus on linear problems. We first consider linear time-independent finite-dimensional systems and show that the optimal controls and states exponentially converge in the transient time (as $T$ tends to infinity) to the ones of the corresponding steady state model. For this to occur suitable observability assumptions need to be imposed. We then extend the results to infinite dimensional systems including the linear heat and wave equations.
\end{abstract}

\section{INTRODUCTION}

In this paper, we address the question of the limiting behavior of optimal control problems as the time-horizon tends to infinity. More specifically, we analyze the convergence of the trajectories and controls which are optimal in $[0, T]$ towards the stationary state and control which are optimal for the corresponding stationary regime.

In general terms, the question could be set as follows. We are given a dynamics

$$
\left\{\begin{array}{l}
x_{t}=f(x, u) \\
x(0)=x_{0}
\end{array}\right.
$$

and a corresponding control problem

$$
\min _{u} J^{T}(u):=\int_{0}^{T} L(x, u) d s, \quad x \text { solution of (1.1), }
$$

under some conditions ensuring that this control problem as well as the stationary analog, namely

are well posed.

$$
\min _{u} J_{s}(u):=L(x, u), \quad \text { with the constraint } f(x, u)=0,
$$

In other words, we assume that both $J^{T}$ and $J_{s}$ admit minimal controls (and states), possibly unique.

The question we address is the following: To which extent the long-horizon optimal controls and states $u^{T}(t), x^{T}(t)$ approximate the stationary ones $\bar{u}, \bar{x}$ as $T \rightarrow \infty$ ?

Of course, this kind of question can lead to several possible directions of investigation (finite or infinite dimensional systems, linear or nonlinear dynamics, generality of the cost functional, etc...). This paper is fully devoted to the linear case, both in finite and infinite dimensions.

The motivation is multifold. On one hand, the study of long time behavior of Mean Fields Games systems in [4], [5]. This leads to a system of PDE which can be seen as the optimality system of a (bilinear) optimal control problem for a convection diffusion equation (see [13]). While studying ergodicity properties and long time stability of the Mean Field Games systems, it is proved in [4], [5] that, in the long horizon problem, the optimal controls and states are close, in average, to the stationary ones and, in some cases,

Partially supported by the ERC Advanced Grant FP7-246775 NUMERIWAVES, the Grant PI2010-04 of the Basque Government, the ESF Research Networking Program OPTPDE and Grant MTM2011-29306 of the MINECO, Spain. 
they are exponentially close in the transient time, namely far from $t=0$ and $t=T$. On the other hand, the use of steady state models for optimal design is classical in aeronautics (see [11]). It is then natural to ask the question of whether these steady state optimal designs are limits as time tends to infinity of time-evolution optimal designs. Of course, in that setting these questions need to be addressed in the complex context of Euler and Navier-Stokes equations and for models of turbulence. The theory is far from being applicable in that context. In [10], motivated by optimal control problems in Fluid Mechanics, a first attempt has been done in the frame of hyperbolic conservation laws, where the problem of passing to the limit as time tends to infinity in the linearization of $1-d$ scalar hyperbolic conservation laws towards the steady state ones has been analyzed.

These results and topics suggest to look more closely to the question of the long time behavior of optimality systems.

One of the main goals of this paper consists in establishing the role played by stabilization and observability estimates in order to get the convergence of finite horizon control problems in $[0, T]$ to their steady state version as $T$ tends to infinity. Indeed, although these assumptions are not needed when considering the optimal control problem in a fixed finite time interval, they are essential to perform the analysis as the time-horizon tends to infinity.

Our first result refers to the finite-dimensional case. We denote by $\mathcal{M}_{N, M}$ the set of $N \times M$ matrices.

Theorem 1.1. Let $A \in \mathcal{M}_{N, N}, B \in \mathcal{M}_{N, M}, u \in L^{2}\left(0, T ; \mathbb{R}^{M}\right)$, and $x_{0} \in \mathbb{R}^{N}$, and consider the dynamics

$$
\left\{\begin{array}{l}
x_{t}+A x=B u \\
x(0)=x_{0} .
\end{array}\right.
$$

Given a matrix $C \in \mathcal{M}_{N, N}$, and some $z \in \mathbb{R}^{N}$, consider the optimal control problem

$$
\min _{u} J^{T}(u)=\frac{1}{2} \int_{0}^{T}\left(|u(t)|^{2}+|C x(t)-z|^{2}\right) d t,
$$

which admits a unique optimal control and state $\left(u^{T}, x^{T}\right)$.

Assuming that $(A, B)$ is controllable and $(A, C)$ is observable, we have that there exist positive constants $\lambda$ and $K$, independent on $T$, such that

$$
\left|u^{T}(t)-\bar{u}\right|+\left|x^{T}(t)-\bar{x}\right| \leq K\left(e^{-\lambda t}+e^{-\lambda(T-t)}\right) \quad \forall t \in[0, T],
$$

where $(\bar{u}, \bar{x})$ are the stationary optimal control and state.

Actually, in this finite-dimensional case, $\lambda$ will be characterized as the exponential stability index for a stabilized dynamics using the algebraic Riccati equation.

Based on the methods we develop to prove this finite dimensional result, we also obtain similar results for parabolic equations (see Theorem 3.10) as well as for wave equations (see Section 4). We pay special attention to the case of control and/or observation operators localized in an open subset of the domain where the dynamics evolves.

Our analysis is restricted to linear problems, but we hope to pave the way for a more general approach to the question which may, eventually, be used in nonlinear problems as well.

Let us also remark that similar questions could as well be addressed from the point of view of Hamilton-Jacobi equations (finite or infinite dimensional). But, although this approach could lead to the convergence analysis of $\frac{1}{T} \min J^{T}$ towards min $J_{s}$ by looking at the long time behavior of the value function, the convergence of the optimal control and states does not seem to be achieved in the literature by this approach. One of the possible reasons is that the construction of the optimal control (and therefore state) involves intrinsically a forward-backward mechanism. This forward-backward feature is evident in the structure of the optimality system and makes the question of long time behavior non standard. As suggested in [4], [5], boundary layers may occurr at $t=0$ and $t=T$ and stability should 
be observed in the transient time. Here we support this hint by showing how, in the linear framework, such result can be obtained using tools from control theory, specifically some stabilization and uncoupling methods as classically addressed in [14], [15]. The exponential rate of this stability is a significant byproduct of our approach, compared with previous ones.

At this stage it is worth mentioning that a whole literature, mainly motivated by economic theories, has been concerned with this kind of stationary behavior in the transient time for long horizon control problems. In that context, such type of result goes under the name of turnpike property (more precisely, middle type turnpike property for our kind of behavior), according to a terminology introduced by Nobel prize P. Samuelson. This property was mostly investigated in the finite dimensional case, as well as in connection to Calculus of Variations, although in a slightly different framework with respect to our setting. Being impossible to quote all relevant contributions in this area, we refer the reader to the extensive survey [21] (and references therein), to [6], [22] for some result on control problems in the infinite dimensional case, and to [7], [9] for some related result on unbounded solutions to the Riccati equation, with applications to zero-sum games.

The rest of this paper is organized as follows. In Section 2 we analyze the finitedimensional case in detail. In Section 3 we discuss the case of parabolic equations. Section 4 is devoted to the wave equation. Finally, in Section 5 we discuss several open problems and related issues.

\section{The Finite Dimensional CASE}

In this section, we consider the finite dimensional case. We denote by $\mathcal{M}_{N, M}$ the set of $N \times M$ matrices, and we consider the dynamics

$$
\left\{\begin{array}{l}
x_{t}+A x=B u \\
x(0)=x_{0}
\end{array}\right.
$$

where $A \in \mathcal{M}_{N, N}, B \in \mathcal{M}_{N, M}$, the control $u \in L^{2}\left(0, T ; \mathbb{R}^{M}\right)$, and $x_{0} \in \mathbb{R}^{N}$. Given a matrix $C \in \mathcal{M}_{N, N}$, and some $z \in \mathbb{R}^{N}$, consider the optimal control problem

$$
\min _{u} J^{T}(u)=\frac{1}{2} \int_{0}^{T}\left(|u(t)|^{2}+|C x(t)-z|^{2}\right) d t .
$$

Note that we could as well add a forcing term $f$ in the dynamics (2.1), however this would not bring any significant novelty to our study, especially for time independent sources $f$. Since this term could be removed up to a translation, eventually changing the values of $x_{0}$ and $z$, there is no loss of generality if we restrict to the case $f=0$.

By standard arguments, problem (2.2) admits a unique optimal control $u^{T}(t)$ in $L^{2}\left(0, T ; \mathbb{R}^{M}\right)$, which satisfies the optimality condition

$$
u^{T}=-B^{*} p^{T}, \quad\left\{\begin{array}{l}
-p_{t}^{T}+A^{*} p^{T}=C^{*}\left(C x^{T}-z\right) \\
p^{T}(T)=0
\end{array}\right.
$$

where $x^{T}$ is the optimal state.

Concerning the dynamics, we assume that

The pair $(A, B)$ is controllable.

It is well known that this is equivalent to require that the matrices $A, B$ satisfy the Kalman rank condition

$$
\operatorname{Rank}\left[B A B A^{2} B \ldots A^{N-1} B\right]=N .
$$

Concerning the cost functional, we assume that

$$
\text { The pair }(A, C) \text { is observable }
$$


which is equivalent to the algebraic condition:

$$
\operatorname{Rank}\left[C C A C A^{2} \ldots C A^{N-1}\right]=N \text {. }
$$

Of course, by duality this means that $\left(A^{*}, C^{*}\right)$ satisfy $(2.5)$, and in particular that $\left(A^{*}, C^{*}\right)$ is controllable. Similarly, assumption (2.4) implies that $\left(A^{*}, B^{*}\right)$ is observable. For the notions of controllability, observability and such dual correspondence in the finite dimensional case, we refer the readers to e.g. [19]. We point out an observability inequality which will be used later.

Remark 2.1. We note that, if $(A, C)$ is observable in the sense of $(2.7)$, then there exists $c$, independent of $T$, such that

$$
|y(T)|^{2} \leq c\left\{\int_{T-1}^{T}|C y|^{2} d t+\int_{T-1}^{T}|f|^{2} d t\right\}
$$

for every $y$ solution of

$$
y_{t}+A y=f \text { in }(0, T)
$$

and every $T \geq 1$.

Indeed, set $\tilde{y}=y-w$, where $w$ satisfies

$$
\left\{\begin{array}{l}
w_{t}+A w=f \quad \text { in }(T-1, T) \\
w(T-1)=0
\end{array}\right.
$$

Then $\tilde{y}$ satisfies the homogeneous equation and, $(A, C)$ being observable, we have

$$
|\tilde{y}(T)|^{2} \leq c \int_{T-1}^{T}|C \tilde{y}|^{2} d t
$$

where $c>0$ is the observability constant in the time interval $[0,1]$. This is a simple and well known fact (see [19] or [24]).

On the other hand, by the well-posedness of the evolution problem, we have

$$
|w(\tau)|^{2} \leq c \int_{T-1}^{\tau}|f|^{2} d t \quad \forall \tau \in[T-1, T]
$$

hence

$$
|y(T)|^{2} \leq 2\left(|\tilde{y}(T)|^{2}+|w(T)|^{2}\right) \leq c\left\{\int_{T-1}^{T}\left[|C y|^{2}+|C w|^{2}\right] d t+|w(T)|^{2}\right\}
$$

which yields (2.8).

Notice that (2.8) also implies the stationary inequality

$$
|z|^{2} \leq \beta\left[|A z|^{2}+|C z|^{2}\right] \quad \forall z \in \mathbb{R}^{N}
$$

for all $z$.

Using (2.9), it is easy to show that there exists a unique optimal state $\bar{x}$, and a unique minimum $\bar{u}$, of the stationary "control problem"

$$
\min _{u} J_{s}(u)=\frac{1}{2}\left(|u|^{2}+|C x-z|^{2}\right), \quad A x=B u,
$$

which is nothing but a constrained minimization problem in $\mathbb{R}^{N}$.

A first basic relation between the evolution and the stationary control problems is the following.

Theorem 2.2. Assume that (2.5) and (2.7) hold true. Then we have

$$
\frac{1}{T} \min _{u \in L^{2}(0, T)} J^{T} \stackrel{T \rightarrow \infty}{\longrightarrow} \min _{u \in \mathbb{R}^{N}} J_{s}
$$


and

$$
\frac{1}{T} \int_{0}^{T}\left(\left|u^{T}(t)-\bar{u}\right|^{2}+\left|C\left(x^{T}(t)-\bar{x}\right)\right|^{2}\right) d t=O\left(\frac{1}{T}\right) \stackrel{T \rightarrow \infty}{\rightarrow} 0 .
$$

In particular, we have

$$
\frac{1}{(b-a) T} \int_{a T}^{b T} x^{T}(t) d t \rightarrow \bar{x} \quad, \quad \frac{1}{(b-a) T} \int_{a T}^{b T} u^{T}(t) d t \rightarrow \bar{u}
$$

for every $a, b \in[0,1], a \neq b$.

Proof. First of all, observe that the stationary optimal control and state $(\bar{u}, \bar{x})$ satisfy

$$
A \bar{x}=B \bar{u}, \quad \bar{u} \cdot v+(C \bar{x}-z) \cdot C \varphi=0 \quad \text { for every } v, \varphi: A \varphi=B v .
$$

In particular, $C^{*}(C \bar{x}-z) \in \operatorname{Ker}(A)^{\perp}$ hence there exist some $\bar{p}$ such that

$$
A^{*} \bar{p}=C^{*}(C \bar{x}-z) .
$$

Note that $\bar{p}$ may not be unique, since it is defined up to elements of $\operatorname{Ker}\left(A^{*}\right)$. However, for any $\bar{p}$ satisfying (2.12), we deduce that

$$
\bar{u} \cdot v+\bar{p} \cdot B v=0 \text { for every } v \text { such that } \exists \varphi: A \varphi=B v
$$

From the optimality systems

we now obtain

$$
\left\{\begin{array}{l}
\left(x^{T}-\bar{x}\right)_{t}+A\left(x^{T}-\bar{x}\right)=B\left(u^{T}-\bar{u}\right) \\
-\left(p^{T}-\bar{p}\right)_{t}+A^{*}\left(p^{T}-\bar{p}\right)=C^{*} C\left(x^{T}-\bar{x}\right)
\end{array}\right.
$$

$\int_{0}^{T}\left|C\left(x^{T}-\bar{x}\right)\right|^{2} d t=\left[\left(x_{0}-\bar{x}\right)\left(p^{T}(0)-\bar{p}\right)\right]+\left[\left(x^{T}(T)-\bar{x}\right) \bar{p}\right]+\int_{0}^{T} B\left(u^{T}-\bar{u}\right)\left(p^{T}-\bar{p}\right) d t$, which yields, using $B^{*} p^{T}=-u^{T}$,

$$
\begin{gathered}
\int_{0}^{T}\left(\left|u^{T}-\bar{u}\right|^{2}+\left|C\left(x^{T}-\bar{x}\right)\right|^{2}\right) d t= \\
=\left[\left(x_{0}-\bar{x}\right)\left(p^{T}(0)-\bar{p}\right)\right]+\left[\left(x^{T}(T)-\bar{x}\right) \bar{p}\right]-\int_{0}^{T} u^{T}\left(\bar{u}+B^{*} \bar{p}\right) d t .
\end{gathered}
$$

Applying inequality (2.8) we have

$$
\left|x^{T}(T)-\bar{x}\right| \leq c\left(\int_{0}^{T}|u-\bar{u}|^{2} d t+\int_{0}^{T}\left|C\left(x^{T}-\bar{x}\right)\right|^{2} d t\right)^{\frac{1}{2}}
$$

for some $c$ independent of $T \geq 1$. Similarly, since $\left(A^{*}, B^{*}\right)$ is observable due to (2.5), we have

$$
\left|\left(p^{T}(0)-\bar{p}\right)\right| \leq c\left(\int_{0}^{T}\left|C\left(x^{T}-\bar{x}\right)\right|^{2} d t+\int_{0}^{T}\left|B^{*}\left(p^{T}-\bar{p}\right)\right|^{2} d t\right)^{\frac{1}{2}},
$$

which implies in particular

$$
\left|\left(p^{T}(0)-\bar{p}\right)\right| \leq c\left(\int_{0}^{T}\left|C\left(x^{T}-\bar{x}\right)\right|^{2} d t+\int_{0}^{T}\left|u^{T}-\bar{u}\right|^{2} d t+\int_{0}^{T}\left|\bar{u}+B^{*} \bar{p}\right|^{2} d t\right)^{\frac{1}{2}}
$$

Using (2.15) and (2.16) in (2.14), we deduce in particular that

$$
\int_{0}^{T}\left(\left|u^{T}-\bar{u}\right|^{2}+\left|C\left(x^{T}-\bar{x}\right)\right|^{2}\right) d t \leq C T
$$

hence the averages $\frac{1}{T} \int_{0}^{T} u^{T}(t) d t$ and $\frac{1}{T} \int_{0}^{T} C x^{T}(t) d t$ are bounded in $\mathbb{R}^{N}$. Moreover, the state equation implies

$$
A\left(\frac{1}{T} \int_{0}^{T} x^{T} d t\right)=\frac{1}{T} \int_{0}^{T} B u^{T} d t-\frac{x^{T}(T)-x_{0}}{T}
$$


and since last term is vanishing as $T \rightarrow \infty$ (because of estimates (2.15) and (2.17)), we deduce that $\frac{1}{T} \int_{0}^{T} A x^{T}(t) d t$ is also bounded. Applying (2.9), we conclude that $\frac{1}{T} \int_{0}^{T} x^{T}(t) d t$ is bounded as well. In addition, we deduce that $A \varphi=B v$ for any $\varphi, v$ such that (possibly for a subsequence) $\frac{1}{T} \int_{0}^{T} x^{T}(t) d t \rightarrow \varphi$ and $\frac{1}{T} \int_{0}^{T} u^{T}(t) d t \rightarrow v$; in particular, this implies, thanks to $(2.13)$, that

$$
\frac{1}{T} \int_{0}^{T} u^{T}\left(\bar{u}+B^{*} \bar{p}\right) d t \stackrel{T \rightarrow \infty}{\rightarrow} 0
$$

for any $\bar{p}$ satisfying (2.12). We conclude from (2.14) that

$$
\frac{1}{T} \int_{0}^{T}\left(\left|u^{T}-\bar{u}\right|^{2}+\left|C\left(x^{T}-\bar{x}\right)\right|^{2}\right) d t \leq \frac{C}{\sqrt{T}}-\frac{1}{T} \int_{0}^{T} u^{T}\left(\bar{u}+B^{*} \bar{p}\right) d t^{T \rightarrow \infty} \rightarrow .
$$

This immediately implies that

$$
\frac{1}{T} \int_{0}^{T} C x^{T}(t) d t \rightarrow C \bar{x} \quad, \quad \frac{1}{T} \int_{0}^{T} u^{T}(t) d t \rightarrow \bar{u}
$$

The convergence of $x^{T}$ to $\bar{x}$ follows now from the averaged state equation and (2.9).

Finally, in a similar way as we did before for $x^{T}$, using now $(2.9)$ for $\left(A^{*}, B^{*}\right)$, we prove that the average $\frac{1}{T} \int_{0}^{T} p^{T} d t$ is bounded in $\mathbb{R}^{N}$ and, up to subsequences, converges to some $\bar{p} \in \mathbb{R}^{N}$. Passing to the limit in the averaged equation

$$
A^{*}\left(\frac{1}{T} \int_{0}^{T} p^{T} d t\right)=\frac{1}{T} \int_{0}^{T} C^{*}\left(C x^{T}-z\right) d t-\frac{p^{T}(0)}{T}
$$

we realize that $\bar{p}$ is a solution of $(2.12)$; and since $u^{T}=-B^{*} p^{T}$, from the convergence of the average we deduce that $\bar{u}=-B^{*} \bar{p}$. We have thus proved that there exists a solution $\bar{p}$ of (2.12) such that $\bar{u}=-B^{*} \bar{p}$. Note that such a $\bar{p}$ is unique since $\left(A^{*}, B^{*}\right)$ is observable. Moreover, going back to (2.14) and (2.15)-(2.16) with this $\bar{p}$, we improve the estimate obtaining

$$
\int_{0}^{T}\left(|u-\bar{u}|^{2}+|C(x-\bar{x})|^{2}\right) d t \leq C
$$

Now we describe more precisely the convergence of $\left(u^{T}, x^{T}\right)$ to $(\bar{u}, \bar{x})$. This is our main result, showing that this convergence holds at exponential rate in the transient time.

Theorem 2.3. Assume that (2.5) and (2.7) hold. Then, there exist positive constants $\lambda$ and $K$, independent on $T$, such that

$$
\left|u^{T}(t)-\bar{u}\right|+\left|x^{T}(t)-\bar{x}\right| \leq K\left(e^{-\lambda t}+e^{-\lambda(T-t)}\right) \quad \forall t \in[0, T] .
$$

Remark 2.4. The rate $\lambda$ is given in the proof of Theorem 2.3; it is the exponential rate of the stabilized dynamics $A+B B^{*} \hat{E}$, where $\hat{E}$ is the solution of the algebraic Riccati equation.

Remark 2.5. It should be not surprising that the assumptions of controllability and observability are crucial in order to have some long time convergence, as for the existence of the infinite horizon problem itself. Without the Kalman rank condition, one or more components of the system may be insensitive to the action of the control (take for instance $A=\left(\begin{array}{cc}-1 & 0 \\ 0 & 0\end{array}\right)$ and $\left.B=\left(\begin{array}{ll}0 & 0 \\ 0 & 1\end{array}\right)\right)$ and therefore cannot be stabilized in any way.

The proof of Theorem 2.3 relies on the fact that the optimal control is actually given by an affine feedback law, which becomes linear when the target is $z=0$, namely when we 
consider:

$$
\begin{aligned}
J_{0}^{T}(u)=\inf \left\{\frac{1}{2} \int_{0}^{T}\left(|u(t)|^{2}+|C x(t)|^{2}\right) d t,\right. \\
\left.\quad u \in L^{2}\left(0, T ; \mathbb{R}^{M}\right), x_{t}+A x=B u, x(0)=x_{0}\right\} .
\end{aligned}
$$

In this case, as it is well known, the feedback law is constructed through the use of the Riccati equation. We recall the following lemma, which can be essentially found in [19] in the case that $C$ is coercive. However, the proof can be easily adapted to the case that $(A, C)$ is observable; we give it here shortly for the reader's convenience, as well as a guideline for the infinite dimensional case, since most of the arguments admit a natural generalization in that setting.

Lemma 2.6. Assume that (2.5) and (2.7) hold. Then, there exists a unique symmetric matrix $\mathcal{E}$, belonging to $C^{1}\left([0, \infty) ; \mathcal{M}_{N, N}\right)$, which solves

$$
\left\{\begin{array}{l}
\mathcal{E}_{t}=C^{*} C-\left(\mathcal{E} A+A^{*} \mathcal{E}\right)-\mathcal{E} B B^{*} \mathcal{E} \quad \text { in }(0,+\infty) \\
\mathcal{E}(0)=0
\end{array}\right.
$$

and $u^{T}(t, x)=-B^{*} \mathcal{E}(T-t) x$ defines an optimal feedback law for (2.20).

Moreover, we have

(i) There exists $M>0$ such that $0<\mathcal{E}(t) \leq M$ for every $t>0$.

(ii) $\mathcal{E}\left(t_{1}\right) \leq \mathcal{E}\left(t_{2}\right)$ for every $t_{1} \leq t_{2}$.

(iii) $\mathcal{E}(t) \stackrel{t \rightarrow \infty}{\rightarrow} \hat{E}$, where $\hat{E}$ is the unique symmetric, positive definite matrix satisfying

$$
\left(\hat{E} A+A^{*} \hat{E}\right)+\hat{E} B B^{*} \hat{E}=C^{*} C .
$$

(iv) The linear system

$$
\left\{\begin{array}{l}
x_{t}+\left(A+B B^{*} \hat{E}\right) x=0 \\
x(0)=x_{0}
\end{array}\right.
$$

is globally asymptotically stable.

Proof. The existence and uniqueness, locally in time, of $\mathcal{E}(t)$ is standard, and clearly it is symmetric.

Moreover, if $\nu(t)$ is the optimal control associated to the minimization problem (2.20), and if $\kappa(t)$ denotes the optimal trajectory, a straightforward computation shows that $\pi(t):=$ $\mathcal{E}(T-t) \kappa(t)$ is the adjoint state.

Since

we deduce

$$
[\kappa \cdot \pi]_{t}=-\left|B^{*} \pi\right|^{2}-|C \kappa|^{2}
$$

$$
\mathcal{E}(T) x_{0} \cdot x_{0}=-\int_{0}^{T}[\mathcal{E}(T-t) \kappa(t) \cdot \kappa(t)]_{t} d t=\int_{0}^{T}\left(\left|B^{*} \pi\right|^{2}+|C \kappa|^{2}\right) d t .
$$

In particular, we have $\mathcal{E}(T) x_{0} \cdot x_{0} \geq 0$, and $\mathcal{E}(T) x_{0} \cdot x_{0}=0$ implies $B^{*} \pi(t)=0$ and $C \kappa(t)=0$ for every $t \in[0, T]$. This means that

$$
\begin{cases}\kappa_{t}+A \kappa=0 & \text { in }(0, T) \\ C \kappa=0 & \text { in }(0, T)\end{cases}
$$

which implies, since $(A, C)$ is observable, that $\kappa(t)=0$ in $(0, T)$, hence $x_{0}=0$. We have proved so far that $\mathcal{E}(T)>0$.

Moreover, we know (since $(A, B)$ is controllable, the system can be steered to zero at any finite time) that there exists some $M>0$, independent on $T$, such that

$$
\inf J_{0}^{T} \leq M\left|x_{0}\right|^{2} \text {. }
$$


We get then from $(2.24)$

$$
\mathcal{E}(T) x_{0} \cdot x_{0}=\inf J_{0}^{T} \leq M\left|x_{0}\right|^{2}
$$

which completes the proof of (i) (since $T$ is arbitrary).

In order to show (ii), let $t_{1} \leq t_{2}$; we just proved that $\mathcal{E}\left(t_{2}\right) x_{0} \cdot x_{0}=\inf J_{0}^{t_{2}}$, corresponding to the optimal control $u_{2}$ and the optimal trajectory $\kappa_{2}$ in $\left[0, t_{2}\right]$, starting from $x_{0}$. Of course we have

$$
\begin{aligned}
\mathcal{E}\left(t_{2}\right) x_{0} \cdot x_{0} & =\inf J_{0}^{t_{2}}=\int_{0}^{t_{2}}\left(\left|u_{2}\right|^{2}+\left|C \kappa_{2}\right|^{2}\right) d t \\
& \geq \int_{0}^{t_{1}}\left(\left|u_{2}\right|^{2}+\left|C \kappa_{2}\right|^{2}\right) d t \geq \inf J_{0}^{t_{1}}=\mathcal{E}\left(t_{1}\right) x_{0} \cdot x_{0}
\end{aligned}
$$

hence $\mathcal{E}\left(t_{2}\right) \geq \mathcal{E}\left(t_{1}\right)$.

Since, for any $x_{0} \in \mathbb{R}^{N}, \mathcal{E}(t) x_{0} \cdot x_{0}$ is increasing and bounded, there exists a matrix $\hat{E}$, symmetric and positive definite, such that $\mathcal{E}(t) \rightarrow \hat{E}$ as $t \rightarrow \infty$. Clearly $\hat{E}$ satisfies (2.22).

Now we show (iv). To this purpose, we prove that $V(x):=\hat{E} x \cdot x$ defines a Lyapunov function for the system (2.23). Indeed, we have

$$
(\hat{E} x(t) \cdot x(t))_{t}=2 \hat{E} x_{t} \cdot x=-2\left(\hat{E} B B^{*} \hat{E} x \cdot x\right)-2 \hat{E} A x \cdot x
$$

and since $2 \hat{E} A x \cdot x=\left(\hat{E} A+A^{*} \hat{E}\right) x \cdot x$, using the equation $(2.22)$ we get

$$
(\hat{E} x(t) \cdot x(t))_{t}=-\left|B^{*} \hat{E} x\right|^{2}-|C x|^{2} .
$$

Therefore, for any $t_{1}<t_{2}$ we have

$$
V\left(x\left(t_{1}\right)\right)-V\left(x\left(t_{2}\right)\right)=\int_{t_{1}}^{t_{2}}\left(\left|B^{*} \hat{E} x\right|^{2}+|C x|^{2}\right) d t \geq 0
$$

and the equality holds if and only if $B^{*} \hat{E} x=C x=0$ in $\left(t_{1}, t_{2}\right)$; since $(A, C)$ is observable, this would imply $x(t)=0$ in $\left(t_{1}, t_{2}\right)$, hence $x\left(t_{1}\right)=x\left(t_{2}\right)=0$. Therefore, $V$ is a strict Lyapunov function for the linear system (2.22), which implies that the system is globally asymptotically stable.

Finally, note that this property would be true for any such matrix $\hat{E}$ satisfying (2.22); therefore (2.25) implies

$$
\hat{E} x_{0} \cdot x_{0}=\int_{0}^{\infty}\left(\left|B^{*} \hat{E} x\right|^{2}+|C x|^{2}\right) d t
$$

and this allows us to deduce that

$$
\hat{E} x_{0} \cdot x_{0}=\inf J_{0}^{\infty}
$$

where $J_{0}^{\infty}$ is the optimal control problem $(2.20)$ with $T=\infty$ (note that this problem makes sense since $(A, B)$ is controllable). Therefore, $(2.27)$ characterizes the positive matrix $\hat{E}$ satisfying (2.22), showing its uniqueness.

Thanks to the above properties, especially due to (iv), we deduce the following:

Corollary 2.7. There exist positive constants $C, \mu>0$ such that

$$
\|\mathcal{E}(t)-\hat{E}\| \leq C e^{-2 \mu t} \quad \forall t>0 .
$$

In particular, $\mu$ can be taken as the exponential rate of the linear system (2.23).

Proof. Set $M=A+B B^{*} \hat{E}$. Subtracting the equations of $\mathcal{E}(t)$ and $\hat{E}$, we notice that the difference satisfies

$$
(\mathcal{E}-\hat{E})_{t}=-\left((\mathcal{E}-\hat{E}) M+M^{*}(\mathcal{E}-\hat{E})\right)-(\mathcal{E}-\hat{E}) B B^{*}(\mathcal{E}-\hat{E}) .
$$


By Lemma 2.6, (iv), we know that $\left\|e^{-t M}\right\| \leq e^{-\mu t}$ for some $\mu>0$. Let us consider the set

$$
\mathcal{X}=\left\{L \in C^{0}\left([0, \infty), \mathcal{M}_{N, N}\right) ;\|L(t)\| \leq \delta e^{-2 \mu t} \forall t>0\right\},
$$

with $\delta>0$ to be made precise later.

Given $L \in \mathcal{X}$, we consider the matrix $Z \in C^{1}([0, \infty))$ which is solution of the differential system

$$
\left\{\begin{array}{l}
Z_{t}=-\left(Z M+M^{*} Z\right)-L B B^{*} L \quad \text { in }(0,+\infty) \\
Z(0)=\Lambda
\end{array}\right.
$$

where $\Lambda$ is some matrix to be fixed later. Since Duhamel's formula implies

$$
Z(t)=e^{-t M^{*}} \Lambda e^{-t M}+\int_{0}^{t} e^{-(t-s) M^{*}} L B B^{*} L e^{-(t-s) M} d s
$$

we have

$$
\|Z(t)\| \leq\|\Lambda\|\left\|e^{-t M}\right\|^{2}+C \delta^{2} e^{-2 \mu t} \leq\left(\|\Lambda\|+C \delta^{2}\right) e^{-2 \mu t} .
$$

If $\|\Lambda\|<\delta / 2$ and $\delta$ is chosen suitably small so that $\delta / 2+C \delta^{2} \leq \delta$, we deduce that $\mathcal{X}$ is an invariant set for the map $\mathcal{T}: L \mapsto Z$. Moreover, as it is easy to check, $\mathcal{T}$ transforms bounded sets into relatively compact sets (in the standard uniform topology). Accordingly, $\mathcal{T}$ admits a fixed point. Choose now $\Lambda=\mathcal{E}\left(t_{0}\right)-\hat{E}$, where $t_{0}$ is chosen so that $\left\|\mathcal{E}\left(t_{0}\right)-\hat{E}\right\|<\delta / 2$, which is possible thanks to Lemma 2.6, (iii). The corresponding fixed point then solves

$$
\left\{\begin{array}{l}
Z_{t}=-\left(Z M+M^{*} Z\right)-Z B B^{*} Z \quad \text { in }(0,+\infty) \\
Z(0)=\mathcal{E}\left(t_{0}\right)-\hat{E}
\end{array}\right.
$$

which is the same equation as (2.28), so we deduce that $\mathcal{E}\left(t+t_{0}\right)-\hat{E}=Z(t)$. Since $Z \in \mathcal{X}$, we conclude that

$$
\|\mathcal{E}(t)-\hat{E}\| \leq \delta e^{-2 \mu\left(t-t_{0}\right)} \forall t>t_{0},
$$

which yields the conclusion.

We are in the position to prove Theorem 2.3.

Proof of Theorem 2.3. $\quad$ For shortness, we drop the dependence on $T$ in the optimal couple $(u(t), x(t))$. Let us set $h(t):=p(t)-\bar{p}-\mathcal{E}(T-t)(x(t)-\bar{x})$, where $\bar{p}$ is the solution of (2.12) such that $\bar{u}=-B^{*} \bar{p}$ (which is given in Theorem 2.2). From the equations of $p$ and (2.21) we have

$$
\begin{aligned}
h_{t} & =(p-\bar{p})_{t}+\mathcal{E}_{t}(T-t)(x-\bar{x})-\mathcal{E}(T-t)(x-\bar{x})_{t} \\
& =A^{*}(p-\bar{p})-A^{*} \mathcal{E}(T-t)(x-\bar{x}) \\
& -\left(\mathcal{E}(T-t) B B^{*} \mathcal{E}(T-t)\right)(x-\bar{x})+\mathcal{E}(T-t) B B^{*}(p-\bar{p})
\end{aligned}
$$

hence $h$ is solution of the linear system

$$
\left\{\begin{array}{l}
h_{t}=\left(A^{*}+\mathcal{E}(T-t) B B^{*}\right) h \quad \text { in }(0, T) \\
h(T)=-\bar{p}
\end{array}\right.
$$

As in the previous Corollary we set $M=A+B B^{*} \hat{E}$; then $h$ solves

$$
h_{t}=M^{*} h+(\mathcal{E}(T-t)-\hat{E}) B B^{*} h
$$

and the Duhamel's formula implies

$$
h(t)=-e^{-(T-t) M^{*}} \bar{p}+\int_{t}^{T} e^{-(s-t) M^{*}}\left((\mathcal{E}(T-s)-\hat{E}) B B^{*} h(s)\right) d s .
$$


Therefore, we get, using Corollary 2.7,

$$
\begin{aligned}
\|h(t)\| & \leq\|\bar{p}\| e^{-\mu(T-t)}+C \int_{t}^{T} e^{-\mu(s-t)} e^{-2 \mu(T-s)}\|h(s)\| d s \\
& \leq C e^{-\mu(T-t)}\left[1+\int_{t}^{T} e^{-\mu(T-s)}\|h(s)\| d s\right],
\end{aligned}
$$

so that

$$
\|h(t)\| \leq C e^{-\mu(T-t)} \quad \forall t \in[0, T],
$$

with $C>0$ independent of $T$.

The equation of $x-\bar{x}$ now reads as

$$
(x-\bar{x})_{t}+\left(A+B B^{*} \hat{E}\right)(x-\bar{x})=B B^{*}(\hat{E}-\mathcal{E}(T-t))(x-\bar{x})-B B^{*} h(t)
$$

hence

$$
x-\bar{x}=e^{-t M}\left(x_{0}-\bar{x}\right)+\int_{0}^{t} e^{-(t-s) M} K(s) d s
$$

where $K(t)=B B^{*}(\hat{E}-\mathcal{E}(T-t))(x-\bar{x})-B B^{*} h(t)$. Using Corollary 2.7 and (2.29) we obtain

$$
\begin{aligned}
\|x(t)-\bar{x}\| & \leq C e^{-\mu t}+\int_{0}^{t} e^{-\mu(t-s)} e^{-2 \mu(T-s)}\|x(s)-\bar{x}\| d s+C \int_{0}^{t} e^{-\mu(t-s)} e^{-\mu(T-s)} d s \\
& \leq C\left(e^{-\mu t}+e^{-\mu(T-t)}\right)+e^{-\mu(T-t)} \int_{0}^{t} e^{-2 \mu(t-s)} e^{-\mu(T-s)}\|x(s)-\bar{x}\| d s
\end{aligned}
$$

which implies that

$$
\|x(t)-\bar{x}\| \leq C\left(e^{-\mu t}+e^{-\mu(T-t)}\right) \quad \forall t \in[0, T]
$$

for some $C>0$.

Recalling that $p-\bar{p}=\mathcal{E}(T-t)(x-\bar{x})+h(t)$, we finally get

$$
\|p(t)-\bar{p}\| \leq C\|x(t)-\bar{x}\|+\|h(t)\| \leq C\left(e^{-\mu t}+e^{-\mu(T-t)}\right),
$$

which of course implies a similar estimate for $u-\bar{u}$.

\section{THE PARABOLIC CASE}

The above finite-dimensional approach can be generalized to infinite dimensional systems in an appropriate functional setting. Following the classical framework given in [14], we consider separately two main situations, corresponding to first or second order time evolutions. In this section we develop the abstract parabolic theory and give an example of application to the case of localized distributed control.

3.1. Abstract setting: Convergence of averages. We assume that $X, H$ are Hilbert spaces such that $X \subset H \subset X^{\prime}$ (with dense embedding), $H$ being identified with its dual.

We assume that $A \in \mathcal{L}\left(X, X^{\prime}\right)$ is a linear continuous operator such that $\mathcal{R}(A)$ is closed $(\mathcal{R}(A)$ and $\mathcal{K} \operatorname{er}(A)$ denote the range and kernel of $A)$. Then we consider:

(i) A control operator $B \in \mathcal{L}\left(U, X^{\prime}\right)$, where $U$ is a Hilbert space.

(ii) An observation operator $C \in \mathcal{L}(X, V)$ going from $X$ into some Hilbert space $V$.

Such assumptions on $B$ and $C$ could be relaxed (see the example given at the end of this Section, when the domain of $C$ is only a dense subset of $X$ ), though a systematic generalization would require the more complex setting of abstract semigroup theory.

Under the above assumptions, the adjoint operators $B^{*}$ and $C^{*}$ are defined and belong to $\mathcal{L}\left(X, U^{\prime}\right)$ and to $\mathcal{L}\left(V^{\prime}, X^{\prime}\right)$ respectively. 
We consider the control problem

$$
\left\{\begin{array}{l}
x_{t}+A x=B u \text { in }(0, T), \\
x(0)=x_{0}
\end{array}\right.
$$

where $u \in L^{2}(0, T ; U)$.

We assume the following conditions on $A$. First of all, we require

$$
\exists \lambda, \mu>0: \quad\langle A x, x\rangle_{X^{\prime}, X}+\mu|x|_{H}^{2} \geq \lambda\|x\|_{X}^{2} \quad \forall x \in X .
$$

Note that $A+\mu I$ is supposed to be coercive at least for large $\mu$ although $A$ itself does not necessarily fulfill the coercivity condition. In particular, $A$ is the generator of a semigroup and the evolution problem

$$
\left\{\begin{array}{l}
x_{t}+A x=f \quad \text { in }(0, T) \\
x(0)=x_{0}
\end{array}\right.
$$

is well posed: for every $x_{0} \in H$ and $f \in L^{2}\left(0, T ; X^{\prime}\right)$ there exists a unique solution $x \in$ $C([0, T] ; H) \cap L^{2}(0, T ; X)$ (see e.g. [14]). The same can be said about the time-evolution control problem with $u \in L^{2}(0, T ; U)$.

We make the following assumption on the evolution system:

Hypothesis 3.1. There exists $\gamma>0$ such that for every $x \in L^{2}(0, T ; X), f \in L^{2}\left(0, T ; X^{\prime}\right)$, $x_{0} \in H$ such that

$$
\left\{\begin{array}{l}
x_{t}+A x=f \quad \text { in }(0, T) \\
x(0)=x_{0}
\end{array}\right.
$$

we have

$$
|x(T)|_{H}^{2} \leq \gamma\left[\int_{0}^{T}\left(\|f\|_{X^{\prime}}^{2}+\|C x\|_{V}^{2}\right) d t+\left|x_{0}\right|_{H}^{2}\right]
$$

for every $T>0$.

Remark 3.2. Let us make a few remarks concerning the previous assumption.

(1) Hypothesis 3.1 holds trivially whenever $A$ is coercive on $X$, i.e. when (3.2) is satisfied with $\mu=0$; in this case (3.4) is satisfied even with $C \equiv 0$. This can be seen by standard energy estimates, taking the scalar product of the equation with $x$ in $H$ and integrating in time.

In general, when $A$ is not coercive, the contribution due to the observation operator $C$ is necessary to have the inequality fulfilled.

(2) Hypothesis 3.1 holds whenever the operators $(A, C)$ satisfy an observability inequality of the type

$$
\left|x\left(T_{0}\right)\right|_{H}^{2} \leq \gamma_{T_{0}}\left[\int_{0}^{T_{0}}\left(\|f\|_{X^{\prime}}^{2}+\|C x\|_{V}^{2}\right) d t\right]
$$

for every $x$ solution of $x_{t}=A x+f$ in $\left(0, T_{0}\right)$. In this case, applying (3.5) in $\left(T-T_{0}, T\right)$ one has

$$
|x(T)|_{H}^{2} \leq \gamma_{1}\left[\int_{T-T_{0}}^{T}\left(\|f\|_{X^{\prime}}^{2}+\|C x\|_{V}^{2}\right) d t\right]
$$

and therefore (3.4) holds for every $T \geq T_{0}$. The local well-posedness of the evolution problem clearly implies (3.4) for $T \leq T_{0}$ as well. 
Notice that, by a trivial superposition principle, and the local well-posedness, (3.5) holds if and only if

$$
\left|x\left(T_{0}\right)\right|_{H}^{2} \leq \tilde{\gamma}_{T_{0}} \int_{0}^{t_{0}}\|C x\|_{V}^{2} d t
$$

for some $\tilde{\gamma}_{T_{0}}>0$, whenever

$$
x_{t}+A x=0 \text { in }\left(0, T_{0}\right) .
$$

This is the standard way in which observability is formulated in the context of controllability problems (see [24]).

(3) Hypothesis 3.1 implies the stationary inequality

$$
\|x\|_{X}^{2} \leq \beta \quad\left[\|A x\|_{X^{\prime}}^{2}+\|C x\|_{V}^{2}\right] \quad \text { for every } x \in X .
$$

Indeed, applying (3.4) to $\tilde{x}:=t x$, for $x \in X$, we get

$$
T^{2}\|x\|_{H}^{2} \leq \gamma \frac{T^{3}}{3}\left[\|A x\|_{X^{\prime}}^{2}+\|C x\|_{V}^{2}\right]+\gamma T\|x\|_{X^{\prime}}^{2} .
$$

Using the embedding of $H$ into $X^{\prime}$ and choosing some $T>\gamma$ we deduce

$$
\|x\|_{H}^{2} \leq \tilde{\gamma}\left[\|A x\|_{X^{\prime}}^{2}+\|C x\|_{V}^{2}\right] .
$$

Due to assumption (3.2), we deduce (3.6).

We also require a similar "observability" estimate for the adjoint state equation.

Hypothesis 3.3. There exists $c_{0}>0$ such that, for every $p \in L^{2}(0, T ; X), f \in L^{2}\left(0, T ; X^{\prime}\right)$, $p_{0} \in H$ such that

we have

$$
\left\{\begin{array}{l}
-p_{t}+A^{*} p=f \quad \text { in }(0, T) \\
p(T)=p_{0}
\end{array}\right.
$$

$$
|p(0)|_{H}^{2} \leq c_{0}\left[\int_{0}^{T}\left|B^{*} p\right|_{U^{\prime}}^{2} d t+\int_{0}^{T}\|f\|_{X^{\prime}}^{2} d t+\left|p_{0}\right|_{H}^{2}\right]
$$

for every $T>0$.

Similar remarks which were done for Hypothesis 3.1 may be adapted and apply for Hypothesis 3.3 too. In particular, Hypothesis 3.3 holds immediately in two main situations, either when $A^{*}$ is coercive, or when $\left(A^{*}, B^{*}\right)$ is observable.

These assumptions can also be written in terms of the stabilizability of the systems involved. In particular, consider the assumption:

Hypothesis 3.4. There exists $L \in \mathcal{L}(X, U)$ and $\delta>0$ such that any solution $x$ of

$$
\left\{\begin{array}{l}
x_{t}+A x=B(L x) \\
x(0)=x_{0}
\end{array}\right.
$$

satisfies

$$
\int_{0}^{T}\|x(t)\|_{X}^{2} d t \leq \delta\left|x_{0}\right|_{H}^{2} \quad \text { for every } T>0
$$

A necessary and sufficient condition for (3.8) to be satisfied is that the corresponding semigroup decays exponentially (see [8]).

We claim that Hypothesis 3.4 implies Hypothesis 3.3. More precisely, the following holds.

Lemma 3.5. If $(A, B)$ is stabilizable, in the sense of Hypothesis 3.4, then Hypothesis 3.3 holds. 
Proof. Take $\varphi \in L^{2}(0, T ; X)$ solution of

$$
\left\{\begin{array}{l}
\varphi_{t}+A \varphi=B(L \varphi) \\
\varphi(0)=\varphi_{0} .
\end{array}\right.
$$

Multiplying by $\varphi$ the equation of $p$ we have

hence

$$
\left(p(0), \varphi_{0}\right)_{H}+\int_{0}^{T}\left\langle p, \varphi_{t}+A \varphi\right\rangle d t=\int_{0}^{T}\langle f, \varphi\rangle d t+\left(p_{0}, \varphi(T)\right)_{H}
$$

$$
\begin{gathered}
\left(p(0), \varphi_{0}\right)_{H}=-\int_{0}^{T}\left\langle B^{*} p, L \varphi\right\rangle d t+\int_{0}^{T}\langle f, \varphi\rangle d t+\left(p_{0}, \varphi(T)\right)_{H} \\
\leq C\left(\int_{0}^{T}\|\varphi\|_{X}^{2} d t\right)^{\frac{1}{2}}\left\{\int_{0}^{T}\left|B^{*} p\right|_{U^{\prime}}^{2} d t+\int_{0}^{T}\|f\|_{X^{\prime}}^{2} d t\right\}^{\frac{1}{2}}+\left(p_{0}, \varphi(T)\right)_{H} \\
\leq C\left|\varphi_{0}\right|_{H}\left\{\int_{0}^{T}\left|B^{*} p\right|_{U^{\prime}}^{2} d t+\int_{0}^{T}\|f\|_{X^{\prime}}^{2} d t\right\}^{\frac{1}{2}}+\left(p_{0}, \varphi(T)\right)_{H} .
\end{gathered}
$$

On the other hand we also have

$$
\begin{gathered}
\frac{1}{2}|\varphi(T)|_{H}^{2}=\frac{1}{2}\left|\varphi_{0}\right|_{H}^{2}+\int_{0}^{T}\langle B(L \varphi)-A \varphi, \varphi\rangle d t \\
\leq \frac{1}{2}\left|\varphi_{0}\right|_{H}^{2}+C \int_{0}^{T}\|\varphi\|_{X}^{2} d t \leq C\left|\varphi_{0}\right|_{H}^{2},
\end{gathered}
$$

hence we conclude

$$
\left(p(0), \varphi_{0}\right)_{H} \leq C\left|\varphi_{0}\right|_{H}\left\{\int_{0}^{T}\left|B^{*} p\right|_{U^{\prime}}^{2} d t+\int_{0}^{T}\|f\|_{X^{\prime}}^{2} d t\right\}^{\frac{1}{2}}+C\left|p_{0}\right|_{H}\left|\varphi_{0}\right|_{H},
$$

and choosing $\varphi_{0}=p(0)$ we deduce (3.7).

In this functional setting and under the above assumptions, we consider the time dependent control problem and the corresponding stationary one. Namely, we consider

$$
\min \left\{J^{T}(u)=\frac{1}{2} \int_{0}^{T}\left(|u(t)|_{U}^{2}+|C x(t)-z|_{V}^{2}\right) d t, u \in L^{2}(0, T ; U), x \text { solves (3.1) }\right\}
$$

and

$$
\min \left\{J_{s}(u)=\frac{1}{2}\left[|u|_{U}^{2}+|C x-z|_{V}^{2}\right], \quad u \in U, x \in X: A x=B u\right\}
$$

where $z \in V$ is a fixed target. Note that $J_{s}$ is defined on the closed subspace

$$
D=\{u \in U: B u \in \mathcal{R}(A)\} .
$$

Thanks to (3.6), the steady state problem is well posed.

Lemma 3.6. The functional $J_{s}$ admits a unique minimizer $\bar{u} \in U$, and there exists a unique state $\bar{x} \in X$ such that $A \bar{x}=B \bar{u}$. Moreover, there exists $\bar{p} \in X$ which satisfies

$$
A^{*} \bar{p}=C^{*} j_{v}(C \bar{x}-z),
$$

$j_{v}$ being the natural inclusion of $V$ into $V^{\prime}$, and we have

$$
(\bar{u}, v)_{U}+\langle\bar{p}, B v\rangle_{X, X^{\prime}}=0, \quad \forall v \in D .
$$

Remark 3.7. The solution of the adjoint equation (3.11) is not unique, since it is only defined up to elements of $\operatorname{Ker}\left(A^{*}\right)$. However, we will show later (see the proof of Theorem 3.8 ) that there exists a unique $\bar{p}$, solution of (3.11), such that $\bar{u}=-j_{u} B^{*} \bar{p}$, being $j_{u}$ the natural inclusion of $U^{\prime}$ into $U$.

This would follow straightforwardly from (3.12) whenever the domain of $J_{s}$ is the whole space $U$, e.g. if $\mathcal{R}(B) \subset \mathcal{R}(A)$. But this may not be the case if, for example, $\operatorname{Ker}\left(A^{*}\right)$ is not empty. Nevertheless, the well-posedness of the steady state control problem (which strongly relies on (3.6)) and the stability of the evolution control problem will allow us to select a solution $\bar{p}$ of (3.11) such that $\bar{u}=-j_{u} B^{*} \bar{p}$. Note that this selection is unique, since $\left(A^{*}, B^{*}\right)$ 
also satisfy (3.6). There may possibly be different ways to construct such a unique adjoint state $\bar{p}$; taking the limit from the evolution control problem, as in our proof of Theorem 3.8 below, is indeed a natural one.

Proof. Due to (3.6) and the constraint $A x=B u$, the level sets $\left\{J_{s}(u) \leq C\right\}$ are bounded in $X$. Therefore, the existence of minima is a standard consequence of weak compactness. By the strict convexity, we have both uniqueness of the minimum $\bar{u}$ and that $C x_{1}=C x_{2}$ for any two possible states associated to $\bar{u}$, i.e. such that $A x_{1}=A x_{2}=B \bar{u}$. It follows from (3.6) that $x_{1}=x_{2}$, hence the optimal state $\bar{x}$ is also unique. Now, the first order optimality condition reads as:

$$
(\bar{u}, v)_{U}+(C \bar{x}-z, C \varphi)_{V}=0
$$

for every $v \in D$ and every $\varphi \in X: A \varphi=B v$.

Let $j_{v}$ denote the inclusion from $V$ into $V^{\prime}$; by taking $v=0$ we deduce that $\left\langle C^{*} j_{v}(C \bar{x}-\right.$ $z), \varphi\rangle_{X^{\prime}, X}=0$ for every $\varphi \in \operatorname{Ker}(A)$, hence $C^{*} j_{v}(C \bar{x}-z) \in \operatorname{Ker}(A)^{\perp}=\mathcal{R}\left(A^{*}\right)$. There exists then an adjoint state $\bar{p} \in X$ satisfying (3.11). We have then, for every $\varphi \in X$ such that $A \varphi=B v$,

$$
(C \bar{x}-z, C \varphi)_{V}=\left\langle A^{*} \bar{p}, \varphi\right\rangle_{X^{\prime}, X}=\langle\bar{p}, B v\rangle_{X, X^{\prime}}
$$

which implies (3.12) (note that this is true for any $\bar{p}$ solution of $(3.11)$ ).

As far as the evolution problem is concerned, there exists a unique optimal control $u^{T} \in$ $L^{2}(0, T ; U)$ and a unique state $x^{T} \in L^{2}(0, T ; X)$, and we have $u^{T}=-j_{u} B^{*} p^{T}$, where $p^{T} \in L^{2}(0, T ; X)$ is the unique solution of

$$
\left\{\begin{array}{l}
-p_{t}^{T}+A^{*} p^{T}=C^{*} j_{v}\left(C x^{T}-z\right) \quad \text { in }(0, T) \\
p^{T}(T)=0 .
\end{array}\right.
$$

As in the finite dimensional case, we have a basic general result on the convergence of the long time averages. Let us point out that similar results can be found in [6], [22] at least for a coercive observation operator.

Theorem 3.8. Assume (3.2) and Hypotheses 3.1 and 3.3. Then we have

$$
\frac{1}{T} \min J^{T} \stackrel{T \rightarrow \infty}{\longrightarrow} \min J_{s}
$$

and

$$
\frac{1}{T} \int_{0}^{T}\left(\left|u^{T}(t)-\bar{u}\right|_{U}^{2}+\left|C\left(x^{T}(t)-\bar{x}\right)\right|_{V}^{2}\right) d t=O\left(\frac{1}{T}\right) \rightarrow 0
$$

Moreover, we have

$$
\frac{1}{(b-a) T} \int_{a T}^{b T} x^{T}(t) d t \rightarrow \bar{x} \quad, \quad \frac{1}{(b-a) T} \int_{a T}^{b T} u^{T}(t) d t \rightarrow \bar{u}
$$

for every $a, b \in[0,1]$.

Proof. Using Hypothesis 3.3 on equation (3.14), and recalling that $u^{T}=-B^{*} p^{T}$, we have

$$
\left|p^{T}(0)\right|_{H}^{2} \leq K\left\{\int_{0}^{T}\left(\left|u^{T}\right|_{U}^{2}+\left|C x^{T}-z\right|_{V}^{2}\right) d t\right\} .
$$

Similarly, for the state equation (3.1), Hypothesis 3.1 and the continuity of $B$ imply

$$
\left|x^{T}(T)\right|_{H}^{2} \leq K\left\{\int_{0}^{T}\left(\left|u^{T}\right|_{U}^{2}+\left|C x^{T}\right|_{V}^{2}\right) d t+\left|x_{0}\right|_{H}^{2}\right\} .
$$


Coupling the equations of $x^{T}$ and $p^{T}$ we deduce

$$
\int_{0}^{T}\left(\left|u^{T}\right|_{U}^{2}+\left|C x^{T}-z\right|_{V}^{2}\right) d t=\left(x_{0}, p^{T}(0)\right)_{H}-\int_{0}^{T}\left(z,\left(C x^{T}-z\right)\right)_{V} d t
$$

so using (3.16) we deduce by now that

$$
\int_{0}^{T}\left(\left|u^{T}\right|_{U}^{2}+\left|C x^{T}-z\right|_{V}^{2}\right) d t \leq C T
$$

and in turn from (3.16) and (3.17):

$$
\left|p^{T}(0)\right|_{H}+\left|x^{T}(T)\right|_{H} \leq C \sqrt{T} .
$$

We deduce from (3.18) and Jensen inequality that $\frac{1}{T} \int_{0}^{T} u^{T} d t$ and $\frac{1}{T} \int_{0}^{T} C x^{T} d t$ are bounded, respectively, in $U$ and in $V$. Since

$$
A\left(\frac{1}{T} \int_{0}^{T} x^{T} d t\right)=\frac{1}{T} \int_{0}^{T} B u^{T} d t+\frac{x_{0}-x^{T}(T)}{T}
$$

appllying (3.6) we get that $\frac{1}{T} \int_{0}^{T} x^{T} d t$ is also bounded in $X$. Similarly we reason for $p^{T}$, obtaining that its average is bounded in $X$.

Now take any $\bar{p}$ satisfying (3.11); subtracting the optimality systems for the evolution and the stationary minima, we have

$$
\left\{\begin{array}{l}
\left(x^{T}-\bar{x}\right)_{t}+A\left(x^{T}-\bar{x}\right)=B\left(u^{T}-\bar{u}\right) \\
-\left(p^{T}-\bar{p}\right)_{t}+A^{*}\left(p^{T}-\bar{p}\right)=C^{*} j_{v} C\left(x^{T}-\bar{x}\right) .
\end{array}\right.
$$

Using that $u^{T}=-B^{*} p^{T}$, we obtain

$$
\begin{gathered}
\int_{0}^{T}\left(\left|u^{T}-\bar{u}\right|^{2}+\left|C\left(x^{T}-\bar{x}\right)\right|^{2}\right) d t= \\
=-\left[\left(x^{T}(T)-\bar{x}\right) \cdot\left(p^{T}(T)-\bar{p}\right)\right]+\left[\left(x_{0}-\bar{x}\right) \cdot\left(p^{T}(0)-\bar{p}\right)\right]-\int_{0}^{T}\left(u^{T},\left(\bar{u}+j_{u} B^{*} \bar{p}\right)\right)_{U} d t .
\end{gathered}
$$

which yields, thanks to (3.19):

$$
\frac{1}{T} \int_{0}^{T}\left(\left|u^{T}-\bar{u}\right|^{2}+\left|C\left(x^{T}-\bar{x}\right)\right|^{2}\right) d t \leq \frac{C}{\sqrt{T}}-\frac{1}{T} \int_{0}^{T}\left(u^{T},\left(\bar{u}+j_{u} B^{*} \bar{p}\right)\right)_{U} d t .
$$

Notice from (3.20), and due to (3.19), that any weak limit of $\frac{1}{T} \int_{0}^{T} u^{T} d t$ will belong to $D=\{u \in U: B u \in \mathcal{R}(A)\}$. Since (3.12) implies $\bar{u}+j_{u} B^{*} \bar{p} \in D^{\perp}$, we have

$$
\lim _{T \rightarrow \infty} \frac{1}{T} \int_{0}^{T}\left(u^{T},\left(\bar{u}+j_{u} B^{*} \bar{p}\right)\right)_{U} d t=0 .
$$

Therefore, we conclude that

$$
\lim _{T \rightarrow \infty} \frac{1}{T} \int_{0}^{T}\left(\left|u^{T}-\bar{u}\right|^{2}+\left|C\left(x^{T}-\bar{x}\right)\right|^{2}\right) d t=0 .
$$

In particular, this identifies the limits of the averages: now we know that $\frac{1}{T} \int_{0}^{T} u^{T} d t$ converges to $\bar{u}$ (strongly in $U$ ) and $\frac{1}{T} \int_{0}^{T} C x^{T} d t$ to $C \bar{x}$ (strongly in $V$ ). Moreover, again from (3.20) and (3.19) we also deduce

$$
\left\|A\left(\frac{1}{T} \int_{0}^{T}\left(x^{T}-\bar{x}\right) d t\right)\right\|_{X^{\prime}}^{2} \leq \frac{c}{T}+\frac{c}{T} \int_{0}^{T}\left\|\left(u^{T}-\bar{u}\right)\right\|_{U}^{2} d t
$$

Therefore, since (3.6) implies

$$
\left\|\frac{1}{T} \int_{0}^{T}\left(x^{T}-\bar{x}\right) d t\right\|^{2} \leq \beta\left\|\frac{1}{T} \int_{0}^{T} A\left(x^{T}-\bar{x}\right) d t\right\|_{X^{\prime}}^{2}+\beta\left\|\frac{1}{T} \int_{0}^{T} C\left(x^{T}-\bar{x}\right) d t\right\|_{V}^{2}
$$


we conclude the convergence of $\frac{1}{T} \int_{0}^{T} x^{T} d t$ to $\bar{x}$. Of course, one can reason similarly for the average in $(a T, b T)$.

Finally, in the same way one can prove that $\frac{1}{T} \int_{0}^{T} p^{T} d t$ converges to some $\bar{p}$ solution of (3.11). Since $u^{T}=-j_{u} B^{*} p^{T}$, taking average and the limit we deduce that $\bar{u}=-j_{u} B^{*} \bar{p}$, thus proving our claim in Remark 3.7.

By using this latter information, observe that the above estimates are all improved; indeed, using now the adjoint state such that $\bar{u}=-j_{u} B^{*} \bar{p},(3.21)$ becomes

$$
\int_{0}^{T}\left(\left|u^{T}-\bar{u}\right|^{2}+\left|C\left(x^{T}-\bar{x}\right)\right|^{2}\right) d t=-\left[\left(x^{T}(T)-\bar{x}\right) \cdot\left(p^{T}(T)-\bar{p}\right)\right]+\left[\left(x_{0}-\bar{x}\right) \cdot\left(p^{T}(0)-\bar{p}\right)\right]
$$

and Hypotheses 3.1 and 3.3 applied to the difference $x^{T}-\bar{x}, p^{T}-\bar{p}$ yield

$$
\begin{gathered}
\int_{0}^{T}\left(\left|u^{T}-\bar{u}\right|^{2}+\left|C\left(x^{T}-\bar{x}\right)\right|^{2}\right) d t \leq K\left\{\left|x^{T}(T)-\bar{x}\right|_{H}+\left|p^{T}(0)-\bar{p}\right|_{H}\right\} \\
\leq K\left\{\int_{0}^{T}\left[\left|u^{T}-\bar{u}\right|_{U}^{2}+\left|C\left(x^{T}-\bar{x}\right)\right|_{V}^{2}\right] d t+1\right\}^{\frac{1}{2}},
\end{gathered}
$$

which implies

$$
\int_{0}^{T}\left(\left|u^{T}-\bar{u}\right|^{2}+\left|C\left(x^{T}-\bar{x}\right)\right|^{2}\right) d t \leq K .
$$

In particular, (3.19) can now be improved and both $p^{T}(t)$ and $x^{T}(t)$ turn out to be bounded in $H$ uniformly in time.

3.2. Exponential convergence: a reference case. As far as the exponential convergence of $\left(u^{T}, x^{T}\right)$ to $(\bar{u}, \bar{x})$ is concerned, a similar strategy as for the finite dimensional case could be followed. As we have shown, this strategy relies on two main steps: the definition and properties of the linear feedback law when the target is $z=0$ (Lemma 2.6) and the exponential estimate given in Corollary 2.7.

The first step is the classical procedure, described e.g. in [14], [15], which allows to uncouple the optimality system, at least in abstract terms. The construction of the feedback law extends in a natural way the finite dimensional case: we consider the control problem (3.9) with $z=0$, and we define an operator on $H$ by setting

$$
\mathcal{E}(T) x_{0}:=p^{T}(0) .
$$

It is immediate to check that $\mathcal{E}(T)$ is linear, continuous and nonnegative, being (see the proof of Theorem 3.8 in case $z=\bar{x}=\bar{p}=0$ ):

$$
\left(\mathcal{E}(T) x_{0}, x_{0}\right)_{H}=\min J_{0}^{T},
$$

where $J_{0}^{T}$ is the functional $J^{T}$ with target $z=0$.

The variational characterization readily implies that $\mathcal{E}\left(t_{1}\right) \leq \mathcal{E}\left(t_{2}\right)$ for every $t_{1} \leq t_{2}$. Since, in addition, $\mathcal{E}(t)$ is uniformly bounded, there exists $\lim _{T \rightarrow \infty}\left(\mathcal{E}(T) x_{0}, x_{0}\right)_{H}$ which is characterized in terms of the regulator problem, defining a limit operator $\hat{E}$. In fact, if $T_{n} \rightarrow \infty$, and we denote $\left(x_{n}, p_{n}\right):=\left(x^{T_{n}}, p^{T_{n}}\right)$ the optimal couple for $J^{T_{n}}$, we know from Theorem 3.8 that

$$
\int_{0}^{T_{n}}\left[\left|B^{*} p_{n}\right|^{2}+\left|C x_{n}\right|^{2}\right] d t \leq K .
$$

So, from Hypotheses 3.1 and $3.3, x_{n}, p_{n}$ are bounded in $H$ uniformly in time, and bounded in $L_{l o c}^{2}(0, T ; X)$ as well. By standard weak compactness, $x_{n}$ and $p_{n}$ converge to a couple $(\hat{x}, \hat{p})$ which satisfies

$$
\int_{0}^{\infty}\left[\left|B^{*} \hat{p}\right|^{2}+|C \hat{x}|^{2}\right] d t \leq K
$$


On one hand, this gives sense to the control problem $J^{\infty}$ (since at least some $u \in L^{2}(0, \infty ; U)$ exists for which the functional is finite). On the other hand, since $\left(x_{n}, p_{n}\right)$ were optimal in $\left(0, T_{n}\right)$, it is easy to see that $(\hat{x}, \hat{p})$ is the unique optimal couple for $J^{\infty}$ and satisfies

$$
\left\{\begin{array}{l}
\hat{x}_{t}+A \hat{x}=-B j_{u} B^{*} \hat{p} \\
-\hat{p}_{t}+A^{*} \hat{p}=C^{*} j_{v} C \hat{x} \\
\hat{x}(0)=x_{0}, \quad \hat{p}(t) \stackrel{t \rightarrow \infty}{\longrightarrow} 0,
\end{array}\right.
$$

where last condition should be meant in weak sense. We set now

$$
\hat{E} x_{0}=\hat{p}(0)
$$

Clearly we have $\hat{E} \in \mathcal{L}(H, H)$, moreover we know that $p_{n}(0)$ converges to $\hat{p}(0)$ weakly in $H$, hence $\mathcal{E}(t) x_{0} \stackrel{t \rightarrow \infty}{\rightarrow} \hat{E} x_{0}$ for any $x_{0} \in H$.

Thus, we have so far defined the feedback laws $\mathcal{E}(t)$ and $\hat{E}$. The semigroup property of the equation implies that the optimal control $u^{T}$ is then defined through the backward feedback

$$
u^{T}(t)=-j_{u} B^{*} \mathcal{E}(T-t) x(t), \quad \forall t \in[0, T] .
$$

Some technical issues may arise here concerning the measurability and continuity of the mapping $t \mapsto \mathcal{E}(t)$, and the meaning itself of the composition operator $B^{*} \mathcal{E}(T-t)$; in principle, $\mathcal{E}$ goes from $H$ into $H$, whereas $B^{*}$ is defined on $X$. To achieve the most generality, such technical issues are to be handled inside the semigroup theory for unbounded operators, a huge literature concerns this delicate issue (see e.g. [2]. [12]).

In order to avoid unnecessary technicalities, we confine here ourselves to the simplest case, assuming that $B \in \mathcal{L}(U, H)$, so $B^{*}$ can be defined directly as the adjoint in $H$. This is typically the case of distributed controls. Moreover, we assume that the embedding $X \subset H$ is compact; in this case, one can easily prove that, for every $x_{0} \in H, \mathcal{E}(t) x_{0}$ is continuous in $[0, \infty)$ and that $\mathcal{E}(t) x_{0} \stackrel{t \rightarrow \infty}{\rightarrow} \hat{E} x_{0}$ strongly in $H$. Now, by the semigroup property, we have $\hat{p}(t)=\hat{E} \hat{x}(t)$ for every $t \in(0, \infty)$, and so $\hat{x}$ is the unique solution of the linear evolution problem

$$
\left\{\begin{array}{l}
x_{t}+\left(A+B j_{u} B^{*} \hat{E}\right) x=0 \\
x(0)=x_{0} .
\end{array}\right.
$$

Note that this system has a unique strong solution, since, under the above conditions, $B j_{u} B^{*} \hat{E}$ is a bounded operator in $H$. Moreover, as in the finite dimensional case, $(\hat{E} x, x)_{H}$ is a Lyapunov function for (3.26); indeed, we have

$$
\frac{d}{d t}\left((\hat{E} \hat{x}(t), \hat{x}(t))_{H}\right)=\frac{d}{d t}\left((\hat{x}(t), \hat{p}(t))_{H}\right)=-\left|B^{*} \hat{E} \hat{x}\right|^{2}-|C \hat{x}|^{2} .
$$

Thanks to $(3.27)$, we can prove that $(3.26)$ is exponentially stable and that $\mathcal{E}(t)$ converges to $\hat{E}$ with exponential rate. We collect these informations in the following result, giving a self contained proof of the exponential convergence only based on Hypotheses 3.1 and 3.3.

Lemma 3.9. Assume that Hypotheses 3.1 and 3.3 hold, and that $X \subset H$ is compact and $B \in \mathcal{L}(U, H)$. Then, the linear system (3.26) is exponentially stable, and there exists $\mu>0$ such that $\|\mathcal{E}(t)-\hat{E}\|_{\mathcal{L}(H, H)} \leq C e^{-\mu t}$, for any $t>0$.

Proof. We first prove the exponential stability of problem (3.26). As before, we denote by $\hat{x}$ the unique strong solution of (3.26), which we also may denote as $e^{-t M} x_{0}$, being $M=A+B j_{u} B^{*} \hat{E}$. 
We start by showing the following observability property, which is consequence of Hypothesis 3.1:

$$
\begin{cases}z_{t}+A z=0 & \text { in }(-\infty, 0), \\ C z=0 & \text { in }(-\infty, 0), \quad \Rightarrow \quad z(t) \equiv 0 \quad \text { in }(-\infty, 0) . \\ z \in L^{\infty}((-\infty, 0) ; H) & \end{cases}
$$

To prove (3.28), we consider $\tilde{z}=e^{\lambda t} z$ and apply (3.4) to $\tilde{z}$ in the interval $(\tau, t)$, obtaining

$$
|\tilde{z}(t)|_{H}^{2} \leq \gamma\left[\lambda^{2} \int_{\tau}^{t}\|\tilde{z}\|_{X^{\prime}}^{2} d s+|\tilde{z}(\tau)|_{H}^{2}\right]
$$

since $C z=0$. Using the embedding $X^{\prime} \subset H$ we get

$$
|\tilde{z}(t)|_{H}^{2} \leq \tilde{\gamma}\left[\lambda^{2} \int_{\tau}^{t}\|\tilde{z}\|_{H}^{2} d s+|\tilde{z}(\tau)|_{H}^{2}\right]
$$

which implies

$$
\int_{\tau}^{t}|\tilde{z}(s)|_{H}^{2} d s \leq \frac{1}{\lambda^{2}} e^{-\tilde{\gamma} \lambda^{2}(\tau-t)}|\tilde{z}(\tau)|_{H}^{2}
$$

Recalling the definition of $\tilde{z}$, and since $z(t)$ is bounded uniformly in $H$, we deduce

$$
\int_{t-1}^{t}|z(s)|_{H}^{2} d s \leq C e^{\left(2 \lambda-\tilde{\gamma} \lambda^{2}\right)(\tau-t)}
$$

Choosing $\lambda$ sufficiently small and letting $\tau \rightarrow-\infty$ we conclude that $z(t)=0$. Thus (3.28) is proved. We claim next that

$$
\sup _{\left|x_{0}\right|_{H} \leq 1}(\hat{E} \hat{x}(t), \hat{x}(t))_{H} \stackrel{t \rightarrow \infty}{\rightarrow} 0
$$

Observe that, due to (3.27), the quantity

$$
l(t):=\sup _{\left|x_{0}\right|_{H} \leq 1}(\hat{E} \hat{x}(t), \hat{x}(t))_{H}
$$

is nonincreasing, hence it admits a limit as $t \rightarrow \infty$. In order to show (3.29), take some $t_{n} \rightarrow \infty$ and $x_{0 n} \in H$ such that $\hat{x}_{n}:=e^{-t M} x_{0 n}$ satisfies

$$
\left(\hat{E} \hat{x}_{n}\left(t_{n}\right), \hat{x}_{n}\left(t_{n}\right)\right)_{H} \geq l\left(t_{n}\right)-\frac{1}{n} .
$$

The sequence $z_{n}(t):=\hat{x}_{n}\left(t+t_{n}\right)$ satisfies

$$
\left(z_{n}\right)_{t}+M z_{n}=0 \quad \text { in }\left(-t_{n},+\infty\right) .
$$

Moreover, due to (3.24) and Hypothesis 3.1, we have $z_{n}$ bounded in $H$ uniformly in time. Since the embedding $X \subset H$ is compact, standard arguments imply that $z_{n}$ is relatively compact in $L^{2}((a, b) ; X)$, and then in $C^{0}([a, b] ; H)$ as well, for any interval $(a, b) \subset \mathbb{R}$. Up to subsequences, $z_{n}(t)$ converges in $H$, pointwise and locally uniformly, to some function $z$ which satisfies

$$
z_{t}+M z=0 \quad \text { in }(-\infty,+\infty)
$$

Moreover, since the definition of $l(t)$ and its monotone character imply

$$
\left(\hat{E} \hat{x}_{n}\left(t_{n}\right), \hat{x}_{n}\left(t_{n}\right)\right)_{H} \leq\left(\hat{E} \hat{x}_{n}\left(t+t_{n}\right), \hat{x}_{n}\left(t+t_{n}\right)\right)_{H} \leq l\left(t+t_{n}\right) \quad \forall t<0,
$$

due to $(3.30)$, we conclude that $(\hat{E} z(t), z(t))_{H}$ is constant in $(-\infty, 0)$ and equal to $\lim _{t \rightarrow \infty} l(t)$. On account of (3.27), we deduce that

$$
B^{*} \hat{E} z=C z=0 \quad \text { in }(-\infty, 0),
$$


and so $z$ solves

$$
\begin{cases}z_{t}+A z=0 & \text { in }(-\infty, 0), \\ C z=0 & \text { in }(-\infty, 0), \\ z \in L^{\infty}((-\infty, 0) ; H) & \end{cases}
$$

hence, by $(3.28), z=0$. This implies that $(\hat{E} z(t), z(t))_{H}=0$, hence $\lim _{t \rightarrow \infty} l(t)=0$, which proves (3.29). Similarly one proves that

$$
\sup _{\left|x_{0}\right|_{H} \leq 1}|\hat{x}(t)|_{H} \stackrel{t \rightarrow \infty}{\rightarrow} 0 .
$$

Indeed, reasoning by contradiction, if (3.31) were not true there would exist $x_{0 n} \in H$ such that $\left|x_{0 n}\right|_{H} \leq 1$, and $t_{n} \rightarrow \infty$ such that $\hat{x}_{n}:=e^{-t M} x_{0 n}$ satisfies $\left|\hat{x}_{n}\left(t_{n}\right)\right|_{H} \geq \varepsilon>0$ for every $n$, for some $\varepsilon>0$. Since, by $(3.29),\left(\hat{E} \hat{x}_{n}\left(t+t_{n}\right), \hat{x}_{n}\left(t+t_{n}\right)\right)_{H} \rightarrow 0$ for any $t$, one can repeat the same reasoning as before on the rescaled function $z_{n}(t)=\hat{x}\left(t+t_{n}\right)$, thus proving that $\left|\hat{x}_{n}\left(t_{n}\right)\right|_{H} \rightarrow 0$ and so obtaining a contradiction. Therefore, (3.31) holds true, which implies the exponential decay of $\hat{x}(t)$. Hence, there exists $\mu>0$ such that

$$
\left|e^{-t M} x_{0}\right|_{H}=|\hat{x}(t)|_{H} \leq e^{-\mu t}\left|x_{0}\right|_{H}
$$

We are left with the estimate of $\|\mathcal{E}(t)-\hat{E}\|$. To this purpose, consider the optimality systems satisfied by $\left(x^{T}, p^{T}\right)$ and $(\hat{x}, \hat{p})$ respectively. Subtracting the equations and integrating in $(0, T)$, one obtains

$$
\int_{0}^{T}\left(\left|B^{*}\left(p^{T}-\hat{p}\right)\right|^{2}+\left|C\left(x^{T}-\hat{x}\right)\right|^{2}\right) d t \leq|\hat{p}(T)|_{H}\left|x^{T}(T)-\hat{x}(T)\right|_{H} .
$$

On the other hand, by Hypothesis 3.1 applied to $x^{T}-\hat{x}$, one has

$$
\left|x^{T}(T)-\hat{x}(T)\right|_{H}^{2} \leq \gamma \int_{0}^{T}\left(\left|B^{*}\left(p^{T}-\hat{p}\right)\right|^{2}+\left|C\left(x^{T}-\hat{x}\right)\right|^{2}\right) d t .
$$

Hence we deduce

$$
\int_{0}^{T}\left(\left|B^{*}\left(p^{T}-\hat{p}\right)\right|^{2}+\left|C\left(x^{T}-\hat{x}\right)\right|^{2}\right) d t \leq C|\hat{p}(T)|_{H}^{2} .
$$

Since $\hat{p}(t)=\hat{E} \hat{x}(t)$, using the above inequality, (3.32) and Hypothesis 3.3, we deduce

$$
\begin{aligned}
&\left|p^{T}(0)-\hat{p}(0)\right|_{H}^{2} \leq C\left\{\int_{0}^{T}\left(\left|B^{*}\left(p^{T}-\hat{p}\right)\right|^{2}+\left|C\left(x^{T}-\hat{x}\right)\right|^{2}\right) d t+|\hat{p}(T)|_{H}^{2}\right\} \\
& \leq C|\hat{p}(T)|_{H}^{2} \leq C e^{-2 \mu T}\left|x_{0}\right|_{H}^{2} .
\end{aligned}
$$

By definition of $\mathcal{E}(T)$ and $\hat{E}$, this yields

$$
\|\mathcal{E}(T)-\hat{E}\|_{\mathcal{L}(H, H)} \leq C e^{-\mu T}
$$

Note that the exponential estimate for $\mathcal{E}(t)-\hat{E}$ obtained in Lemma 3.9 is less accurate than what we found in Corollary 2.7 in the finite dimensional case. Indeed, the estimate in Lemma 3.9 is obtained through only energy estimates relying on Hypotheses 3.1 and 3.3, without an explicit use of the differential Riccati equation. An alternative would have been to proceed again as in Corollary 2.7, namely through a fixed point argument, which however would require the existence and uniqueness of solutions to the differential Riccati equation. Under the assumptions made, this is still possible using the results known in the literature (see e.g. [2], [12] and references therein), although it requires a more delicate functional setting. On the other hand, the conclusion of Lemma 3.9, obtained with self contained arguments, is enough in order to deduce the exponential stability for the control problem $(3.9)$. 
Theorem 3.10. Assume that Hypotheses 3.1 and 3.3 hold, and that $X \subset H$ is compact and $B \in \mathcal{L}(U, H)$. Then, the optimal control $u^{T}$ of (3.9) is given by the affine law

$$
u^{T}=\bar{u}-j_{u} B^{*}\left[\mathcal{E}(T-t)\left(x^{T}(t)-\bar{x}\right)+h^{T}(t)\right]
$$

where $h^{T}$ solves the evolution problem

$$
\begin{cases}-h_{t}+\left(A^{*}+\mathcal{E}(T-t) B j_{u} B^{*}\right) h=0 & \text { in }(0, T) \\ h(T)=-\bar{p} & .\end{cases}
$$

and there exists $K, \mu>0$ such that we have

$$
\left\|x^{T}(t)-\bar{x}\right\|_{H}+\left\|u^{T}(t)-\bar{u}\right\|_{H} \leq K\left(e^{-\mu t}+e^{-\mu(T-t)}\right) \quad \forall t \in[0, T] .
$$

Remark 3.11. The constant $\mu$ is the exponential rate of the stabilized dynamics (3.26), given by Lemma 3.9. The constant $K$ only depends on $x_{0}$ and on the target $z$, eventually through the stationary states $\bar{x}, \bar{p}$, as well as on the operators $A, B, C$, through the constants in Hypotheses 3.1, 3.3.

Proof. Let $h^{T}$ denote the solution of (3.33). As in the proof of Theorem 2.3, one can deduce from Lemma 3.9 and Gronwall's lemma that $\left|h^{T}(t)\right|_{H} \leq C e^{-\mu(T-t)}$ for $t \in(0, T)$. Now, by the only definition of the feedback $\mathcal{E}(t)$, one can see that

$$
p^{T}(t)-\bar{p}=\mathcal{E}(T-t)\left(x^{T}(t)-\bar{x}\right)+h^{T}(t) .
$$

Indeed, this is just verifying a duality argument; for any $\varphi \in H$, we have

$$
\left(p^{T}(t)-\bar{p}, \varphi\right)_{H}=\left(x^{T}(t)-\bar{x}, \mathcal{E}(T-t) \varphi\right)_{H}+\left(h^{T}(t), \varphi\right)_{H}
$$

as it is readily seen by using $(3.33)$ and recalling that $q(t):=\mathcal{E}(T-t) \varphi$ is the adjoint state solving

$$
\begin{cases}-q_{t}+A^{*} q=C^{*} j C z & \text { in }(t, T) \\ z_{t}+A z=-B j_{u} B^{*} q & \text { in }(t, T) \\ z(t)=\varphi, \quad q(T)=0 & \end{cases}
$$

Once the affine feedback law (3.34) is given, one can go back to the equation satisfied by $x^{T}-\bar{x}$; using the exponential estimates for $\mathcal{E}(T-t)-\hat{E}$ as well as for $h^{T}$, and the exponential decay of $A+B j_{u} B^{*} \hat{E}$, the conclusion follows as in Theorem 2.3.

As a model example of the previous abstract results, we may consider the Dirichlet problem for a parabolic equation with internal control and observation. In a bounded domain $\Omega \subset \mathbb{R}^{N}$, let us consider the equation

$$
\begin{cases}y_{t}-\operatorname{div}(M(x) \nabla y)+c(x) y+B(x) \cdot \nabla y=u \chi_{\omega} & \text { in } \Omega \times(0, T) \\ y=0 & \text { on }(0, T) \times \partial \Omega \\ y(0)=y_{0} \in L^{2}(\Omega), & \end{cases}
$$

where $M(x) \in L^{\infty}\left(\Omega, \mathbb{R}^{N} \times \mathbb{R}^{N}\right)$ satisfies (a.e. in $\left.\Omega\right) \lambda I \leq M(x) \leq \Lambda I$ for some $\lambda, \Lambda>0$, and where $c(x) \in L^{\infty}(\Omega), c(x) \geq 0$, and $B(x) \in L^{\infty}(\Omega)^{N}$ (though we could assume some more general integrability condition).

Then, consider the associated control problem

$$
\min J^{T}(u)=\frac{1}{2} \int_{0}^{T}\left[|u(t)|_{L^{2}(\omega)}^{2}+|y(t)-z|_{L^{2}\left(\omega_{0}\right)}^{2}\right] d t,
$$

where $u \in L^{2}\left(0, T ; L^{2}(\omega)\right)$ and $y$ solves (3.35). Here $\omega$ and $\omega_{0}$ are two open subsets of $\Omega$.

Setting $X=H_{0}^{1}(\Omega), X^{\prime}=H^{-1}(\Omega)$ and $H=L^{2}(\Omega)$, and

$$
A(y)=-\operatorname{div}(M(x) \nabla y)+c(x) y+B(x) \cdot \nabla y
$$

we have $A \in \mathcal{L}\left(H_{0}^{1}(\Omega), H^{-1}(\Omega)\right)$, and actually $A$ is an isomorphism, since we have

$$
\|y\|_{X} \leq K\|A y\|_{X^{\prime}} \text {. }
$$


Moreover, observe that, since $A$ has positive first eigenvalue (as consequence of maximum principle, see e.g. [3]), both $A$ and $A^{*}$ are exponentially stable, in particular Hypothesis 3.3 and 3.1 would hold for any choice of the control operator $B$ and the observation operator $C$. In the suggested example, we have taken $U=L^{2}(\omega)$ and $V=L^{2}\left(\omega_{0}\right)$. Then, we deduce from Theorem 3.10 the following stability result.

Corollary 3.12. Let us consider the control problem (3.36) and let $\left(u^{T}, y^{T}\right)$ be the optimal control and state. Then, there exists $\lambda>0$ such that

$$
\left\|y^{T}(t)-\bar{y}\right\|_{L^{2}(\Omega)}+\left\|u^{T}(t)-\bar{u}\right\|_{L^{2}(\Omega)} \leq K\left(e^{-\lambda t}+e^{-\lambda(T-t)}\right) \quad \forall t \in[0, T],
$$

where $\bar{u}$ and $\bar{y}$ are the optimal control and state of the corresponding stationary control problem.

In the above example, $C$ is the restriction operator to $\omega_{0}$ and, in particular, it is a bounded operator. But it may happen $C$ to be defined on some domain $D(C)$ which is dense in $X$ but, eventually, $C$ not to be continuous in the topology of $X$. A typical situation of this kind may happen when the observation is taken on boundary traces. In this case, it is not possible to define the adjoint state $\bar{p}$ by using $C^{*}$ and (3.11) or (3.14). However, as in [14], one can often define the adjoint state through a duality or transposition argument as follows.

Assume that there is some space $M \subset X$ such that $C \in \mathcal{L}(M, V)$ and, at the same time, that $A$ is an isomorphism from $M$ into $H$ and $B \in \mathcal{L}(U, H)$. In this case the adjoint state $\bar{p}$ can be defined by the equation

$$
\bar{p} \in H, \quad(\bar{p}, A \varphi)_{H}=(C \bar{x}-g, C \varphi)_{V} \quad \forall \varphi \in M .
$$

We argue similarly for the evolution problem. We define

$$
W:=\left\{\varphi \in L^{2}(0, T ; M): \varphi_{t}+A \varphi \in L^{2}(H), \varphi(0)=0\right\}
$$

and then, as before, there exists a unique $p \in L^{2}(0, T ; H)$ such that

$$
\int_{0}^{T}\left(p, \varphi_{t}+A \varphi\right)_{H} d t=\int_{0}^{T}(C x-g, C \varphi)_{V} d t \quad \text { for every } \varphi \in W
$$

and we have $u=-j_{u} B^{*} p$.

One can modify now Hypothesis 3.3 as follows: $\exists c_{0}>0$ such that, for every $f \in$ $L^{2}(0, T ; V), p_{0} \in H$, and any solution $p$ of

$$
\left\{\begin{array}{l}
p \in L^{2}(0, T ; H), \\
\int_{0}^{T}\left(p, \varphi_{t}+A \varphi\right)_{H} d t=\int_{0}^{T}(f, C \varphi)_{V} d t+\left(p_{0}, \varphi(T)\right)_{H} \quad \text { for every } \varphi \in W,
\end{array}\right.
$$

we have

$$
|p(t)|_{H}^{2} \leq c_{0}\left[\int_{0}^{T}\left|B^{*} p\right|_{U^{\prime}}^{2} d t+\int_{0}^{T}\|f\|_{V}^{2} d t+\left|p_{0}\right|_{H}^{2}\right] \quad \text { for a.e. } t \in(0, T)
$$

where $B^{*}$ denotes the adjoint of $B$ as a mapping from $L^{2}(0, T ; U)$ to $L^{2}(0, T ; H)$.

Under such premises, we can extend Theorem 3.8 to this setting too. The only variation to the previous proof consists in the use of the optimality system, which now requires (3.37) and (3.38). In this case we get

$$
\int_{0}^{T}\left(p-\bar{p}, \varphi_{t}+A \varphi\right)_{H} d t=\int_{0}^{T}(C(x-\bar{x}), C \varphi)_{V} d t-(\bar{p}, \varphi(T))_{H}
$$

for every $\varphi \in W$. Then (3.39) implies

$$
|p(t)-\bar{p}|_{H}^{2} \leq c_{0}\left[\int_{0}^{T}\left|B^{*}(p-\bar{p})\right|_{U^{\prime}}^{2} d t+\int_{0}^{T}\|C(x-\bar{x})\|_{V}^{2} d t+|\bar{p}|_{H}^{2}\right] .
$$


Let $\xi_{\varepsilon}(t)=\min (t / \varepsilon, 1)$, and observe that

$$
\left(\xi_{\varepsilon}(x-\bar{x})\right)_{t}+A\left(\xi_{\varepsilon}(x-\bar{x})\right)=\xi_{\varepsilon} B(u-\bar{u})+\frac{1}{\varepsilon}(x-\bar{x}) \chi_{(0, \varepsilon)} .
$$

In particular $\xi_{\varepsilon}(x-\bar{x}) \in W$ and we have, using (3.40),

$$
\begin{aligned}
& \int_{0}^{T} \xi_{\varepsilon}(p-\bar{p}, B(u-\bar{u}))_{H} d t+\int_{0}^{\varepsilon} \frac{1}{\varepsilon}(p-\bar{p},(x-\bar{x}))_{H} d t \\
& =\int_{0}^{T} \xi_{\varepsilon}(C(x-\bar{x}), C(x-\bar{x}))_{V} d t-(\bar{p}, x(T)-\bar{x})_{H} .
\end{aligned}
$$

Denoting $o(1)$ quantities which converge to zero as $\varepsilon \rightarrow 0$, we deduce that

$$
\int_{0}^{T}|u-\bar{u}|_{U}^{2} d t+\int_{0}^{T}|C(x-\bar{x})|_{V}^{2} d t=\int_{0}^{\varepsilon} \frac{1}{\varepsilon}(p-\bar{p},(x-\bar{x}))_{H} d t+(\bar{p}, x(T)-\bar{x})_{H}+o(1) .
$$

Since (3.41) implies

$$
\begin{gathered}
\frac{1}{\varepsilon} \int_{0}^{\varepsilon}(p-\bar{p},(x-\bar{x}))_{H} d t \leq \\
\leq c_{0}\left(\frac{1}{\varepsilon} \int_{0}^{\varepsilon}|x-\bar{x}|^{2} d t\right)^{\frac{1}{2}}\left[\int_{0}^{T}\left|B^{*}(p-\bar{p})\right|_{U^{\prime}}^{2} d t+\int_{0}^{T}\|C(x-\bar{x})\|_{V}^{2} d t+|\bar{p}|_{H}^{2}\right]^{\frac{1}{2}}
\end{gathered}
$$

by letting $\varepsilon \rightarrow 0$ we obtain

$$
\begin{gathered}
\int_{0}^{T}|u-\bar{u}|_{U}^{2} d t+\int_{0}^{T}|C(x-\bar{x})|_{V}^{2} d t \leq \\
\leq c_{0}\left|x_{0}-\bar{x}\right|_{H}\left[\int_{0}^{T}|u-\bar{u}|_{U}^{2} d t+\int_{0}^{T}\|C(x-\bar{x})\|_{V}^{2} d t+|\bar{p}|_{H}^{2}\right]^{\frac{1}{2}}+(\bar{p}, x(T)-\bar{x})_{H} .
\end{gathered}
$$

Using Hypothesis 3.1 to estimate the last term, we conclude as in Theorem 3.8.

As an example, the above setting can be used with the control problem (3.35) where the observation operator is taken at the boundary: $C y=\frac{\partial y}{\partial \nu}$. In this case we have $X=H_{0}^{1}(\Omega)$ and $M=H^{2}(\Omega) \cap H_{0}^{1}(\Omega)$.

\section{The WAVE EQUATION}

4.1. The abstract setting. We now consider in a similar way the case of second order problems in time, namely

$$
\left\{\begin{array}{l}
y_{t t}+A y+F y_{t}=B u \quad \text { in }(0, T) \\
y(0)=y_{0}, y_{t}(0)=y_{1}
\end{array}\right.
$$

We assume that $X, H, A$ satisfy the same basic assumptions as before $\left(X \subset H \subset X^{\prime}\right.$, $A \in \mathcal{L}\left(X, X^{\prime}\right)$ has closed range and satisfies (3.2)). In addition, we now assume that $A$ is symmetric.

As far as the control and the observation operators are concerned, for simplicity we assume that $B \in \mathcal{L}(U, H)$ and $C \in \mathcal{L}(X, V)$, where $U, V$, are Hilbert spaces. We also assume that $F \in \mathcal{L}(H, H)$.

Under these assumptions, for $\left(y_{0}, y_{1}\right) \in X \times H$ and $u \in L^{2}(0, T ; U)$, problem (4.1) admits a unique solution $y \in C^{0}([0, T] ; X) \cap C^{1}([0, T] ; H)$. We refer e.g. to $[14$, Chapter IV, Thm 1.1]. We then consider

$$
\min \left\{J(u)=\frac{1}{2} \int_{0}^{T}\left[|u(t)|_{U}^{2}+|C y(t)-g|_{V}^{2}\right] d t, \quad u \in L^{2}(0, T ; U)\right\}
$$

where $y$ is the solution of (4.1).

In this context, we replace Hypothesis 3.1 by the following one.

Hypothesis 4.1. There exists $\gamma>0$ such that

$$
|y(T)|_{H}^{2}+\left\|y_{t}(T)\right\|_{X^{\prime}}^{2} \leq \gamma\left[\int_{0}^{T}\left(\|f\|_{X^{\prime}}^{2}+\|C y\|_{V}^{2}\right) d t+\left|y_{0}\right|_{X}^{2}+\left|y_{1}\right|_{H}^{2}\right]
$$


for every $f \in L^{2}(0, T ; H)$, every $y_{0} \in X, y_{1} \in H$, and every $y$ solution of

$$
\left\{\begin{array}{l}
y_{t t}+A y+F y_{t}=f \\
y(0)=y_{0}, y_{t}(0)=y_{1}
\end{array}\right.
$$

Similarly as in the parabolic case, Hypothesis 4.1 implies the validity of the stationary inequality (3.6). To see this fact, one can take $y=t^{2} x$ in the previous inequality obtaining

$$
T^{4}|x|_{H}^{2}+4 T^{2}\|x\|_{X^{\prime}}^{2} \leq \gamma \int_{0}^{T}\left[\left(t^{4}\|A x\|_{X^{\prime}}^{2}+t^{4}\|C x\|_{V}^{2}\right)+4\|x\|_{X^{\prime}}^{2}+\|F(2 t x)\|_{X^{\prime}}^{2}\right] d t .
$$

Using that $H \subset X^{\prime}$, and so $\|F(2 t x)\|_{X^{\prime}}^{2} \leq c t^{2}|x|_{H}^{2}$, and choosing $T$ sufficiently large, one deduces inequality (3.6).

Finally, Hypothesis 3.3 may be replaced by

Hypothesis 4.2. There exists $C>0$ such that

$$
\|p(0)\|_{H}^{2}+\left\|p_{t}(0)\right\|_{X^{\prime}}^{2} \leq C\left(\left|p_{T}\right|_{X}^{2}+\int_{0}^{T}\left\|B^{*} p\right\|_{U^{\prime}}^{2} d t+\int_{0}^{T}\|f\|_{X^{\prime}}^{2} d t\right)
$$

for every $f \in L^{2}(0, T ; H)$, every $p_{T} \in X$, and every $p$ solution of

$$
\left\{\begin{array}{l}
p_{t t}+A p-F^{*} p_{t}=f \\
p(T)=p_{T}, p_{t}(T)=0
\end{array}\right.
$$

As in the parabolic case, instead of the previous assumption, one could alternatively ask for the existence of a stabilizing feedback.

Hypothesis 4.3. There exists $L_{1} \in \mathcal{L}(X, U), L_{2} \in \mathcal{L}(H, U)$ and $\delta, \mu>0$ such that any solution $y$ of

$$
\left\{\begin{array}{l}
y_{t t}+A y+F y_{t}=B\left(L_{1} y+L_{2} y_{t}\right) \\
y(0)=y_{0}, y_{t}(0)=y_{1}
\end{array}\right.
$$

satisfies

$$
\int_{0}^{T}\left[\|y\|_{X}^{2}+\left|y_{t}\right|_{H}^{2}\right] d t \leq \delta\left[\left\|y_{0}\right\|_{X}^{2}+\left|y_{1}\right|_{H}^{2}\right] \quad \forall T>0
$$

for every given $y_{0} \in X, y_{1} \in H$.

Again, we stress that Hypothesis 4.3 implies Hypothesis 4.2.

Lemma 4.4. If $(A, B)$ is stabilizable, in the sense of Hypothesis 4.3, then Hypothesis 4.2 holds.

Proof. By duality we get

$$
\left.\left(p_{t}-F^{*}(p), y\right)_{H}\right|_{0} ^{T}-\left.\left(p, y_{t}\right)_{H}\right|_{0} ^{T}+\int_{0}^{T}\left\langle p, y_{t t}+A y+F y_{t}\right\rangle d t=\int_{0}^{T}(f, y)_{H} d t
$$

Let $y$ solve (4.4). Then

$$
\left|\int_{0}^{T}\left\langle p, y_{t t}+A y+F y_{t}\right\rangle d t\right| \leq C\left(\int_{0}^{T}\left\|B^{*} p\right\|_{U^{\prime}}^{2} d t\right)^{\frac{1}{2}}\left[\left\|y_{0}\right\|_{X}^{2}+\left|y_{1}\right|_{H}^{2}\right]^{\frac{1}{2}} .
$$

Moreover, we have

$$
\begin{aligned}
|y(T)|_{H}^{2}+\left|y_{t}(T)\right|_{H}^{2} \leq & C \int_{0}^{T}\left[\|y(t)\|_{X}^{2}+\left|y_{t}\right|_{H}^{2}\right] d t+\left|y_{0}\right|_{H}^{2}+\left|y_{1}\right|_{H}^{2} \\
& \leq C\left[\left\|y_{0}\right\|_{X}^{2}+\left|y_{1}\right|_{H}^{2}\right]
\end{aligned}
$$


so that

$$
\begin{gathered}
\left|\left(p(0), y_{1}\right)_{H}-\left(p_{t}(0)-F^{*} p(0), y_{0}\right)_{H}\right| \leq \\
\leq C\left(\left|p_{T}\right|_{H}^{2}+\int_{0}^{T}\left\|B^{*} p\right\|_{U^{\prime}}^{2} d t+\int_{0}^{T}\|f\|_{X^{\prime}}^{2} d t\right)^{\frac{1}{2}}\left[\left\|y_{0}\right\|_{X}^{2}+\left|y_{1}\right|_{H}^{2}\right]^{\frac{1}{2}} .
\end{gathered}
$$

If we choose $y_{0}=0$ and $y_{1}=p(0)$, we deduce

$$
\|p(0)\|_{H}^{2} \leq C\left(\left|p_{T}\right|_{H}^{2}+\int_{0}^{T}\left\|B^{*} p\right\|_{U^{\prime}}^{2} d t+\int_{0}^{T}\|f\|_{X^{\prime}}^{2} d t\right)
$$

and next we estimate $p_{t}(0)$ obtaining (4.3).

Hypotheses 4.1 and 4.2 are enough to deduce the long time convergence in average.

Theorem 4.5. Assume (3.2) and Hypotheses 4.1 and 4.2. Then the conclusions of Theorem 3.8 hold true.

Proof. The proof is similar to that of Theorem 3.8. For simplicity, we drop the dependence on $T$ for the optimal evolution pair $\left(u^{T}(t), x^{T}(t)\right)$. The optimality system now takes the form $^{1}$

$$
\left\{\begin{array}{l}
(x-\bar{x})_{t t}+A(x-\bar{x})+F\left(x_{t}\right)=B(u-\bar{u}) \\
u-\bar{u}=-j_{u} B^{*}(p-\bar{p}) \\
(p-\bar{p})_{t t}+A(p-\bar{p})-\left(F^{*}(p)\right)_{t}=C^{*} j_{v} C(x-\bar{x})
\end{array}\right.
$$

where $\bar{p}$ is the unique adjoint state satisfying (3.11) and such that $\bar{u}=-j_{u} B^{*} \bar{p}$ (see Remark 3.7). We have

$$
\begin{gathered}
\int_{0}^{T}|C(x-\bar{x})|_{V} d t-\int_{0}^{T}(B(u-\bar{u}), p-\bar{p})_{H} d t= \\
-\left.\left(F^{*}(p-\bar{p}), x-\bar{x}\right)_{H}\right|_{0} ^{T} \\
+\left.\left((p-\bar{p})_{t},(x-\bar{x})\right)_{H}\right|_{0} ^{T}-\left((x-\bar{x})_{t},(p-\bar{p})\right)_{H}{\underset{0}{\mid}}_{0}^{T} .
\end{gathered}
$$

Using Hypotheses 4.1 and 4.2 we estimate the right hand side and we obtain

$$
\int_{0}^{T}|C(x-\bar{x})|_{V}^{2} d t+\int_{0}^{T}|u-\bar{u}|_{U}^{2} d t \leq C
$$

which proves (3.15).

Let us now deduce the convergence in average for the optimal states. First of all, using either Hypothesis 4.1 or Hypothesis 4.2, the previous bound implies that $|x(t)|_{H}$ and $\left\|x_{t}(t)\right\|_{X^{\prime}}$ are bounded, as well as $|p(t)|_{H}$ and $\left\|p_{t}(t)\right\|_{X^{\prime}}$. From the equation we have

$$
\int_{0}^{T} A(x-\bar{x})=\int_{0}^{T} B(u-\bar{u}) d t-\int_{0}^{T} F\left(x_{t}\right) d t+\left[x_{1}-x_{t}(T)\right]
$$

the equality being valid in $X^{\prime}$. Since

$$
\int_{0}^{T} F\left(x_{t}\right) d t=F \int_{0}^{T} x_{t} d t=F\left(x(T)-x_{0}\right),
$$

the continuity of $F$ in $H$ and the bound established before imply

Therefore we have

$$
\left|\int_{0}^{T} F\left(x_{t}\right) d t\right|_{H} \leq c \text {. }
$$

$$
\left\|\frac{1}{T} \int_{0}^{T} A(x-\bar{x})\right\|_{X^{\prime}} \leq \frac{c}{T}+\frac{1}{T} \int_{0}^{T}\|B(u-\bar{u})\|_{X^{\prime}} d t \leq \frac{c}{T}+\frac{c}{T} \int_{0}^{T}\|u-\bar{u}\|_{U} d t .
$$

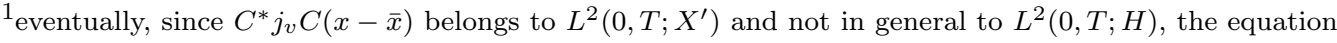
of the adjoint state should be defined by transposition. This is however a minor detail, see also [14].
} 
Using (3.6), we get

$$
\left\|\frac{1}{T} \int_{0}^{T}(x-\bar{x}) d t\right\|_{X}^{2} \leq \beta\left\{\left\|\frac{1}{T} \int_{0}^{T} A(x-\bar{x})\right\|_{X^{\prime}}^{2}+\left\|\frac{1}{T} \int_{0}^{T} C(x-\bar{x})\right\|_{V}^{2}\right\}
$$

and then

$$
\left\|\frac{1}{T} \int_{0}^{T}(x-\bar{x}) d t\right\|_{X}^{2} \leq \frac{c}{T^{2}}+\frac{c}{T} \int_{0}^{T}\|u-\bar{u}\|_{U}^{2} d t+\frac{1}{T} \int_{0}^{T}\|C(x-\bar{x})\|_{V}^{2} d t \leq \frac{c}{T},
$$

which gives the convergence in mean of the optimal trajectories. The same argument can be applied in any interval $(a T, b T)$, with $0 \leq a<b \leq 1$. With a similar reasoning on the dual state equation, the same conclusion also holds for $p-\bar{p}$.

4.2. Examples. As examples, we consider two control problems for the wave equation, with or without damping, with localized distributed control.

Example 4.6. Consider the damped wave equation

$$
\begin{cases}y_{t t}-\Delta y+y_{t}=u \chi_{\omega} & \text { in }(0, T) \times \Omega \\ y=0 & \text { on }(0, T) \times \partial \Omega \\ y(0)=y_{0}, y_{t}(0)=y_{1} . & \end{cases}
$$

where $\omega \subset \Omega$ as before, and consider the internal observation $C y=y \chi_{\omega_{0}}, \omega_{0} \subset \Omega$. Thanks to the damping term, Hypothesis 4.1 is satisfied (even with $C=0$ ) and so is Hypothesis 4.3 with $L_{1}=L_{2}=0$ (and therefore Hypothesis 4.2 holds too).

Example 4.7. Here we consider the pure wave equation

$$
\begin{cases}y_{t t}-\Delta y=u \chi_{\omega} & \text { in }(0, T) \times \Omega \\ y=0 & \text { on }(0, T) \times \partial \Omega \\ y(0)=y_{0}, y_{t}(0)=y_{1}, & \end{cases}
$$

with again the internal observation $C y=y \chi_{\omega_{0}}$. If we assume that $\omega, \omega_{0}$ satisfy the so-called Geometric Control Condition (GCC) (see [24]), then Hypotheses 4.1 and 4.3 are satisfied.

Indeed, recall that if a subset $\mathcal{O} \subset \Omega$ verifies the GCC, then the solution of

$$
\begin{cases}y_{t t}-\Delta y+y_{t} \chi_{\mathcal{O}}=0 & \text { in }(0, T) \times \Omega \\ y=0 & \text { on }(0, T) \times \partial \Omega \\ y(0)=y_{0}, y_{t}(0)=y_{1}, & \end{cases}
$$

satisfies

$$
E(t):=\int_{\Omega}\left[y_{t}(t)^{2}+|\nabla y(t)|^{2}\right] d x \leq C e^{-\mu t} E(0) \quad \forall t>0
$$

for some $\mu>0$.

In particular, if $\omega$ satisfies the GCC, then Hypothesis 4.3 holds true with $L_{2}\left(y_{t}\right)=-y_{t}$ and $L_{1}=0$, and therefore Lemma 4.4 applies to $(A, B)$. On the other hand, since $\omega_{0}$ also satisfies the GCC, $\left(A, C^{*}\right)$ is also stabilizable in the sense of Hypothesis 4.3 , and so, by Lemma 4.4, $(A, C)$ satisfies Hypothesis 4.1.

Moreover, in both Examples 4.6 and 4.7 the convergence rate is exponential, namely there exist $\lambda>0$ and $K>0$ such that

$$
\left\|y^{T}(t)-\bar{y}\right\|_{H_{0}^{1}(\Omega)}+\left\|u^{T}(t)-\bar{u}\right\|_{L^{2}(\Omega)} \leq K\left(e^{-\lambda t}+e^{-\lambda(T-t)}\right) \quad \forall t \in[0, T],
$$

where $\left(y^{T}, u^{T}\right)$ and $(\bar{y}, \bar{u})$ are the optimal trajectories and controls for the wave equation and for the stationary problem, respectively. The proof of (4.10) follows the same strategy as for Theorem 3.10, suitably adapted to the hyperbolic problem. In particular, one first considers 
the linear feedback defined for the target $z=0$, which corresponds to the optimality system (written in abstract form)

$$
\begin{cases}y_{t t}^{T}+A y^{T}+F\left(y_{t}^{T}\right)=-B j_{u} B^{*} q^{T} & t \in(0, T) \\ q_{t t}^{T}+A q^{T}-\left(F^{*}\left(q^{T}\right)\right)_{t}=C^{*} j_{v} C y^{T} & t \in(0, T) \\ y^{T}(0)=y_{0}, y_{t}^{T}(0)=y_{1}, \quad q^{T}(T)=0, q_{t}^{T}(T)=0 . & \end{cases}
$$

This defines an operator $\mathcal{E}(T)$ on $H_{0}^{1}(\Omega) \times L^{2}(\Omega)$ by setting $\mathcal{E}(T)\left(y_{0}, y_{1}\right):=\left(F^{*}\left(q^{T}(0)\right)-\right.$ $\left.q_{t}^{T}(0), q^{T}(0)\right)$. In particular, the range of $\mathcal{E}(T)$ is included in $\left(H_{0}^{1}(\Omega) \times L^{2}(\Omega)\right)^{\prime}$ and $\mathcal{E}(T)$ is a nonnegative, uniformly bounded operator from $H_{0}^{1}(\Omega) \times L^{2}(\Omega)$ into its dual, as well as monotone with respect to $T$, since

$$
\left\langle\mathcal{E}(T)\left(y_{0}, y_{1}\right),\left(y_{0}, y_{1}\right)\right\rangle=\left(F^{*}\left(q^{T}(0)\right)-q_{t}^{T}(0), y_{0}\right)_{H}+\left(q^{T}(0), y_{1}\right)_{H}=\min J_{0}^{T},
$$

where $J_{0}^{T}$ is the cost functional with target $z=0$. Thanks to the monotone character and the uniform bound of $\mathcal{E}$, and using local compactness properties of solutions of the wave equation, the operator $\mathcal{E}(T)$ is proved to have a limit $\hat{E}$ such that $\hat{E}\left(y_{0}, y_{1}\right)=\left(F^{*}\left(\hat{q}^{T}(0)\right)-\right.$ $\left.\hat{q}_{t}(0), \hat{q}(0)\right)$, where $\hat{q}$ is the adjoint state for the regulator problem, i.e. the problem with infinite horizon. Then, as in the parabolic case, the exponential decrease for the regulator problem is established using the quadratic form defined by $\hat{E}$ as Lyapunov functional and the conclusion follows using the Hypotheses 4.1 and 4.2. Let us note that in Examples 4.6 and 4.7, such hypotheses hold in a strong form, since $y(t)$ and $y_{t}(t)$ are uniformly estimated in $H_{0}^{1}(\Omega)$ and in $L^{2}(\Omega)$ respectively, while (4.2) and (4.3) only require, a priori, an estimate for weaker norms. This makes the proof of the exponential stability (4.10) easier to achieve, following the same approach as in the previous section apart from suitable adaptation of the energy estimates.

4.3. Slow convergence. Let us conclude by observing that a different decay estimate in (4.9) may yield similar convergence results although affecting the rate of convergence. To be more precise, we recall that whenever $\mathcal{O}$ is a general open nonempty subset of $\Omega$, not necessarily satisfying the GCC, then the energy satisfies a log-decay, namely we have for the solution of (4.8) (see [17], [23] and [16] for an extension to wave equations with nonlinear damping)

$$
\int_{\Omega}\left[y_{t}(t)^{2}+|\nabla y(t)|^{2}\right] d x \leq \frac{C_{0}}{[\log (2+t)]^{2}}\left(\left\|y_{0}\right\|_{H^{2}}^{2}+\left\|y_{1}\right\|_{H^{1}}^{2}\right) \quad \forall t>0 .
$$

Therefore, proceeding by duality as in Lemma 4.4, any solution $p$ of

satisfies

$$
\left\{\begin{array}{l}
p_{t t}-\Delta p=f \\
p(T)=p_{T}, p_{t}(T)=0
\end{array}\right.
$$

$$
\begin{aligned}
& \|p(0)\|_{H^{-1}(\Omega)}^{2}+\left\|p_{t}(0)\right\|_{\left(H_{0}^{1} \cap H^{2}(\Omega)\right)^{\prime}}^{2} \\
& \quad \leq C \frac{T}{[\log (2+T)]^{2}}\left(\left\|p_{T}\right\|_{L^{2}(\Omega)}^{2}+\int_{0}^{T} \int_{\mathcal{O}}|p|^{2} d x d t+\int_{0}^{T}\|f\|_{L^{2}(\Omega)}^{2} d t\right),
\end{aligned}
$$

which is a weaker version of (4.3). Nevertheless, if we start in (4.7) with initial data $y_{0} \in$ $H^{2} \cap H_{0}^{1}(\Omega)$ and $y_{1} \in H_{0}^{1}(\Omega)$, we can still follow the proof of Theorem 4.5 obtaining now (note that $\bar{p} \in H^{2}(\Omega)$ by regularity of the Laplace operator)

$$
\begin{aligned}
& \int_{0}^{T} \int_{\omega_{0}}|y-\bar{y}|^{2} d x d t+\int_{0}^{T} \int_{\omega}|u-\bar{u}|^{2} d x d t \\
& \quad \leq C \frac{T}{[\log (2+T)]^{2}}\left(\left\|y_{0}\right\|_{H^{2}}^{2}+\left\|y_{1}\right\|_{H^{1}}^{2}+\|\bar{p}\|_{H^{2}}^{2}\right) .
\end{aligned}
$$

Thanks to (4.12), we deduce the convergence of the average with a rate $O\left(\frac{1}{[\log (T)]^{2}}\right)$. 


\section{FURTHER COMMENTS AND OPEN PROBLEMS}

- The control problem for the evolution equation

$$
x_{t}+A x=f \quad \text { in }(0, T)
$$

can be reduced to a fixed time interval $(0,1)$ by a simple change in the time variable $t=T s$ so that the state equation becomes

$$
\frac{1}{T} \tilde{x}_{t}+A \tilde{x}=f \quad \text { in }(0,1) .
$$

But this does not seem to simplify the problems under consideration.

The singular limit behavior of parabolic-parabolic systems towards parabolicelliptic systems of this kind has been recently analyzed in the context of nullcontrollability in [1].

- Our analysis is restricted to linear problems. The same questions arise in the nonlinear context as well. As mentioned in the introduction, in the theory of Mean Field Games these questions arise for bilinear problems, while, in aeronautics, they emerge naturally in the nonlinear models of Fluid Mechanics. A systematic analysis of these issues is to be developed. Some results for nonlinear models exist with respect to the turnpike property (see [21]); it would be natural to analyze how the results of this paper extend to the nonlinear setting, e.g. to semilinear heat and wave equations.

- In the nonlinear context it is also natural to address the same issues for the linearized equations around the optimal states and controls so to analyze the proximity of steady state sensitivity with respect to the time-evolution one.

- As indicated in the introduction it would be worth trying to obtain the results in this paper from the point of view of Hamilton-Jacobi equations (finite or infinite dimensional).

- In the heat and wave equations considered in this paper the control entered in the system in a localized manner, in an open subset of the domain where the equation evolves. But often in control theoretical applications the control enters through the boundary and the same can occur to the observation operator. Although most of the techniques employed in this paper can be adapted to that case, special attention has to be paid to the unboundedness of the control and observation operators and the details have to be worked out in each specific setting either in the general context of abstract semigroups or in specific examples using transposition. We refer for instance to [12], [20] for a systematic presentation of the abstract theory of observation and control of infinite-dimensional systems.

Acknowledgement. We warmly thank Emmanuel Trélat for useful discussions on the topic of this article. We also thank the editors and referees for fruitful suggestions, in particular concerning the connection of our work with the turnpike property in the mathematical economics theory.

A. Porretta wishes to thank the BCAM Center in Bilbao for the invitation and for providing a stimulating environment for collaboration.

\section{REFERENCES}

[1] M. Bendahmane and F. W. Chaves-Silva, Null controllability of a degenerated reaction-diffusion system in cardiac electro-physiology, C. R. Acad. Sci. Paris, 350 (2012), no. 11-12, 587-590.

[2] A. Bensoussan, G. Da Prato, M. Delfour, S.K. Mitter, Representation and Control of Infinite Dimensional Systems, Second edition. Systems \& Control: Foundations \& Applications. Birkhäuser Boston, Inc., Boston, MA, 2007.

[3] H. Berestycki, L. Nirenberg, S.R.S. Varadhan, The principal eigenvalue and maximum principle for second-order elliptic operators in general domains, Comm. Pure Appl. Math. 47 (1994), no. 1, 47-92.

[4] P. Cardaliaguet, J.-M. Lasry, P.-L. Lions, A. Porretta, Long time average of Mean Field Games, Netw. Heterog. Media 7 (2012), no. 2, 279-301. 
[5] P. Cardaliaguet, J.-M. Lasry, P.-L. Lions, A. Porretta, Long time average of Mean Field Games in case of nonlocal coupling, preprint.

[6] D.A. Carlson, A. Haurie, A. Jabrane, Existence of overtaking solutions to infinite dimensional control problems on unbounded time intervals, SIAM J Control Optim 25 (1987), 1517-1541.

[7] G. Da Prato, M.C. Delfour, Unbounded solutions to the linear quadratic control problem, SIAM J. Control Optim. 30 (1992), 31-48.

[8] R. Datko, Uniform Asymptotic Stability of Evolutionary Processes in a Banach Space, SIAM J. Math. Anal., 3(3) (1972), 428-445.

[9] M.C. Delfour, O. Dello Sbarba, Linear quadratic differential games: closed loop saddle points, SIAM J. Control Optim. 47 (2008), 3138-3166.

[10] M. Ersoy, E. Feireisl and E. Zuazua, Sensitivity analysis of $1-d$ steady forced scalar conservation laws, preprint.

[11] A. Jameson, Optimization Methods in Computational Fluid Dynamics (with Ou, K.), Encyclopedia of Aerospace Engineering, Edited by Richard Blockley and Wei Shyy, John Wiley \& Sons, Ltd., ISBN: 978-0-470-68665-2, DOI: 10.1002/9780470686652, 2010.

[12] I. Lasiecka, R. Triggiani, Control theory for partial differential equations, Vol. I \& II, Encyclopedia of Mathematics and its Applications, Cambridge University Press (1999).

[13] J.-M. Lasry, P.-L. Lions, Jeux à champ moyen. II. Horizon fini et contròle optimal. C. R. Math. Acad. Sci. Paris 343 (2006), no. 10, 679-684.

[14] J.-L. Lions. Optimal control of systems governed by partial differential equations. Springer-Verlag, New York-Berlin, 1971.

[15] J.-L. Lions, Exact controllability, stabilization and perturbations for distributed systems, SIAM Review 30 (1988), n. 1, 1-68.

[16] K. D. Phung, Decay of solutions of the wave equation with localized nonlinear damping and trapped rays, Mathematical control and Related Fields, 1 (2) (2011), 251-265.

[17] L. Robbiano, Fonction de cô̂t et contrôle des solutions des équations hyperboliques, Asymptotic Anal. 10 (1995), 95-115.

[18] D.L. Russell, Controllability and stabilizability theory for linear partial differential equations: recent progress and open questions, SIAM Review 20 (1978), n. 4, 639-739.

[19] E. Trélat, Contrôle optimal. Théorie \& applications. Mathématiques Concrètes. Vuibert, Paris, 2005.

[20] M. Tucsnak, G. Weiss, Observation and Control for Operator Semigroups, Birkhäuser, 2009.

[21] A. J. Zaslavski, Turnpike properties in the calculus of variations and optimal control. Nonconvex Optimization and its Applications, 80. Springer, New York, 2006.

[22] A. J. Zaslavski, Existence and Structure of Optimal Solutions of Infinite-Dimensional Control Problems, Appl Math Optim 42 (2000), 291-313.

[23] X. Zhang and E. Zuazua, Long time behavior of a coupled heat-wave system arising in fluid-structure interaction, Archive for Rational Mechanics and Analysis, 184 (1) (2007), 49-120.

[24] E. Zuazua, Controllability and Observability of Partial Differential Equations: Some results and open problems, in Handbook of Differential Equations: Evolutionary Equations, vol. 3, C. M. Dafermos and E. Feireisl eds., Elsevier Science, 2006, pp. 527-621.

Alessio Porretta

Università di Roma Tor Vergata

Dipartimento di MatematicA

VIA DELLA RICERCA SCIENTIFICA 1

00133 Roma, ITALY

E-mail address: porretta@mat.uniroma2.it

ENRIQUE ZUAZUA

BCAM - Basque Center for Applied Mathematics,

Alameda Mazarredo, 14

E-48009 Bilbao Basque Country Spain

Ikerbasque, Basque Foundation for Science,

Alameda UrquiJo 36-5, Plaza Bizkaia

48011 Bilbao Basque Country Spain

E-mail address: zuazua@bcamath.org 